\title{
Variational quantum algorithm for estimating the quantum Fisher information
}

\author{
Jacob L. Beckey $\oplus^{1,2,3}$ M. Cerezo, ${ }^{1,4,5}$ Akira Sone, ${ }^{1,4,5,6}$ and Patrick J. Coles ${ }^{1,5}$ \\ ${ }^{1}$ Theoretical Division, Los Alamos National Laboratory, Los Alamos, New Mexico 87544, USA \\ ${ }^{2}$ JILA, NIST, and University of Colorado, Boulder, Colorado 80309, USA \\ ${ }^{3}$ Department of Physics, University of Colorado, Boulder, Colorado 80309, USA \\ ${ }^{4}$ Center for Nonlinear Studies, Los Alamos National Laboratory, Los Alamos, New Mexico 87544, USA \\ ${ }^{5}$ Quantum Science Center, Oak Ridge, Tennessee 37931, USA \\ ${ }^{6}$ Aliro Technologies, Inc., Boston, Massachusetts 02135, USA
}

(Received 8 January 2021; revised 23 June 2021; accepted 14 January 2022; published 2 February 2022)

\begin{abstract}
The quantum Fisher information (QFI) quantifies the ultimate precision of estimating a parameter from a quantum state and can be regarded as a reliability measure of a quantum system as a quantum sensor. However, estimation of the QFI for a mixed state is in general a computationally demanding task. In this paper we present a variational quantum algorithm called variational quantum Fisher information estimation (VQFIE) to address this task. By estimating lower and upper bounds on the QFI, based on bounding the fidelity, VQFIE outputs a range in which the actual QFI lies. This result can then be used to variationally prepare the state that maximizes the QFI, for the application of quantum sensing. In contrast to previous approaches, VQFIE does not require knowledge of the explicit form of the sensor dynamics. We simulate the algorithm for a magnetometry setup and demonstrate the tightening of our bounds as the state purity increases. For this example, we compare our bounds with literature bounds and show that our bounds are tighter.
\end{abstract}

DOI: 10.1103/PhysRevResearch.4.013083

\section{INTRODUCTION}

The goal of quantum sensing is to utilize quantum coherence or quantum entanglement to better estimate unknown parameters of quantum systems via measurement [1-3]. This includes quantum magnetometry [4,5], quantum thermometry [6-9], quantum illumination [10,11], distributed sensing [12,13], and quantum system identification [14] for the estimation of the Hamiltonian parameters [15-19], graph structure [20], or system dimensions [21,22]. The methodologies developed in quantum sensing are expected to contribute to the progress in various state-of-the-art fields of science and technology, such as molecule structure determination $[23,24]$, biosensing $[25,26]$, nanomaterial magnetism $[27,28]$, dark matter detection [29], and gravitational wave detection [30,31].

Quantum Fisher information (QFI) is a fundamentally important quantity in quantum sensing because it quantifies the ultimate precision achievable in estimating a parameter $\theta$ from a quantum state $\rho_{\theta}$ via the quantum Cramér-Rao bound (QCRB) [32,33]. For single-parameter estimation, QFI is associated with the standard fidelity between the true state $\rho_{\theta}$ and an error state $\rho_{\theta+\delta}$. The intuition behind this relation is that QFI captures the response of the quantum state to

Published by the American Physical Society under the terms of the Creative Commons Attribution 4.0 International license. Further distribution of this work must maintain attribution to the author(s) and the published article's title, journal citation, and DOI. a small change in $\theta$. A true state with a high QFI will be very distinguishable from the error state, making it easier to estimate the parameter via measurement.

While the QFI has been extensively studied for pure quantum states [34,35], the case of mixed states has received considerably less attention, with recent theoretical results shedding light on using mixed states for metrology applications [36]. Since preparing pure quantum states is intrinsically a difficult task (due to system-environment interactions), most quantum states prepared in quantum hardware are mixed states. As such, one of the main goals for state-of-the-art technologies for quantum control is the reduction of quantum noise to be able to prepare states with high purities.

An example of such technologies is quantum computers. While quantum devices are expected to outperform classical computers in many tasks such as factoring and simulating complex systems, currently available quantum devices, known as noisy intermediate-scale quantum (NISQ) computers [37], are prone to hardware noise and hence prepare mixed states. Moreover, the limited number of qubits and constrained circuit depth make it impractical to implement error correction schemes. This does not preclude, however, the possibility of still employing mixed states (with either high purity or high rank) for practical applications, and this is precisely the scope of this work.

Variational quantum algorithms (VQAs) are one of the most promising strategies to overcome these limitations in the NISQ era [38] and make use of near-term quantum devices. In VQAs, a cost function $C(\boldsymbol{\alpha})$ is efficiently estimated with a quantum computer, while part of the computational complexity is pushed to a classical optimizer which minimizes the 
cost by adjusting the parameters $\boldsymbol{\alpha}$ of a parametrized quantum circuit. VQAs have been studied for various applications [39-54], and the scaling of their trainability has been explored recently [55-61].

In the past decade, there have been several proposals to apply classical machine learning methods to the quantum parameter estimation problem [62-64]. However, even more recently, the prospect of instead using NISQ devices to enhance quantum sensing capabilities has become an exciting research direction and is precisely the topic of our work. State preparation for sensing via VQAs has been proposed for phase estimation in trapped atomic arrays $[65,66]$, noisy magnetometry [67], and multiple-parameter estimation [68] and for phase estimation assisted by purity-loss measurement $[69,70]$. However, it is not obvious that these protocols avoid an important practical issue known as barren plateaus in the cost training landscape [55,56]. In addition, they can also require detailed information about the dynamics of how the parameter $\theta$ is encoded in the quantum system, which is not always known in practice, and which is not needed in this paper.

Here, we propose a VQA to estimate the QFI on mixed states that addresses the issues previously mentioned. Namely, our method could avoid barren plateaus and does not require information about the dynamics of interest (i.e., the explicit mathematical form of the generator of unitary dynamics is not required). We name this algorithm the variational quantum Fisher information estimation (VQFIE) algorithm. VQFIE computes upper and lower bounds on the QFI, and these bounds are based on bounding the quantum fidelity. We specifically focus on bounds obtained by truncating the spectrum of the exact state [45], which can be computed by taking advantage of previous variational methods for obtaining a quantum state's principal components [48]. One can then use VQFIE to variationally prepare the state that maximizes the estimated QFI. As schematically shown in Fig. 1, we expect applications of our proposed algorithm in various fields such as materials science, biology, and chemistry.

The paper is organized as follows. In Sec. II, we formulate the basic theory for VQFIE by introducing lower and upper bounds on the QFI. Then, in Sec. III we present the structure of the VQFIE algorithm for computing the aforementioned bounds and for estimating the QFI. In Sec. IV, we present numerical simulations of VQFIE for a magnetometry application. We finally compare our bound with the literature in Sec. V, followed by the concluding remarks in Sec. VI.

\section{THEORETICAL FRAMEWORK}

\section{A. General background}

Consider the scenario where an $n$-qubit quantum state $\rho$, known as the probe state, interacts with a source that encodes the information of a single parameter $\theta$ and maps the input state into the so-called exact state $\rho_{\theta}$. The QFI quantifies the ultimate precision $\Delta \theta$ when estimating the parameter $\theta$ from $\rho_{\theta}$ via the quantum Cramér-Rao bound as [32,33]

$$
(\Delta \theta)^{2} \geqslant \frac{1}{\nu I\left(\theta ; \rho_{\theta}\right)}
$$

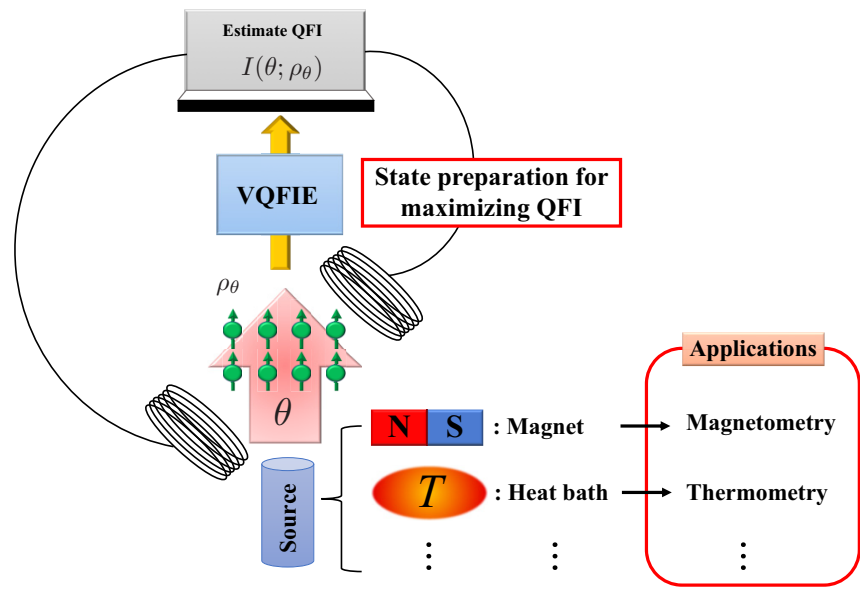

FIG. 1. Application of the variational quantum Fisher information estimation (VQFIE) algorithm. A quantum system $\rho$ interacts with a source that encodes the information of a parameter $\theta$ in the state as $\rho_{\theta}$. The goal of VQFIE is to estimate the quantum Fisher information (QFI), $I\left(\theta ; \rho_{\theta}\right)$, which is related to the minimal achievable uncertainty when estimating $\theta$ from $\rho_{\theta}$. One can then use VQFIE to variationally prepare the state $\rho$ that maximizes the estimated QFI. The VQFIE algorithm can then be used in many applications related to precision sensing, such as magnetometry and thermometry.

where $v$ is the number of measurement repetitions used to estimate $\theta$ and where $I\left(\theta ; \rho_{\theta}\right)$ is the QFI which is uniquely determined by the parameter to be estimated and the measured quantum state. We note that the standard mathematical definition of the QFI is given in terms of a so-called symmetric logarithmic derivative (SLD) operator, which need not be unique in general [71]. However, here we utilize a more experimentally useful definition of the QFI for single-parameter estimation, given as [32,33]

$$
I\left(\theta ; \rho_{\theta}\right)=-4 \lim _{\delta \rightarrow 0} \partial_{\delta}^{2} F\left(\rho_{\theta}, \rho_{\theta+\delta}\right),
$$

where $F(\rho, \sigma)=\left\|\sqrt{\rho_{\theta}} \sqrt{\rho_{\theta+\delta}}\right\|_{1}=\operatorname{Tr}\left[\sqrt{\sqrt{\rho_{\theta}} \rho_{\theta+\delta} \sqrt{\rho_{\theta}}}\right]$ is the standard fidelity between the exact state $\rho_{\theta}$ and the error state $\rho_{\theta+\delta}$.

Equation (2) quantifies the sensitivity of the state $\rho$ to small changes $\delta$ in the parameter as the second partial derivative of the fidelity between exact and error states. Hence the more sensitive $\rho_{\theta}$ is to these small $\theta$ changes, the larger the QFI is, and the more precise the estimation of the parameter will be according to Eq. (1). Note that here no assumptions were made regarding what $\theta$ is, or how it was encoded in $\rho_{\theta}$. This formalism then encompasses cases such as $\theta$ being the magnitude of a field (magnetometry) or a temperature (thermometry).

In practice, one approximates the QFI by

$$
I_{\delta}\left(\theta ; \rho_{\theta}\right)=8 \frac{1-F\left(\rho_{\theta}, \rho_{\theta+\delta}\right)}{\delta^{2}},
$$

where $|\delta| \ll 1$. Although a smaller $\delta$ will always lead to a better approximation, the achievable range of values will depend on the experimental implementation. In the limit of the parameter shift $\delta$ approaching zero, Eq. (3) becomes the QFI as $I\left(\theta ; \rho_{\theta}\right)=\lim _{\delta \rightarrow 0} I_{\delta}\left(\theta, \rho_{\theta}\right)$, because the fidelity takes its maximum at $\delta=0[32,33]$. For pure states such a quantity can 
be efficiently computed on a quantum computer, as the fidelity between two pure states is simply given by their overlap, i.e., $F\left(\left|\psi_{\theta}\right\rangle,\left|\psi_{\theta+\delta}\right\rangle\right)=\left|\left\langle\psi_{\theta} \mid \psi_{\theta+\delta}\right\rangle\right|$. However, for a general mixed state there is no efficient algorithm to directly compute the standard fidelity and the QFI in (3). This does not preclude the possibility of estimating the QFI by calculating efficiently computable upper and lower bounds of $I_{\delta}\left(\theta ; \rho_{\theta}\right)$, which is precisely the goal of VQFIE.

\section{B. Bounds on the quantum Fisher information}

There are many ways in which to bound and estimate the QFI [66,69,70,72-74]; however, many have scaling that precludes their implementation on near-term devices. Moreover, most known methods require detailed knowledge of how the unknown parameter was encoded in the state, which is certainly not always known in practice. For a VQA intended to run on NISQ hardware, quantities of interest must be computable with sufficiently shallow quantum circuits. In this section we present such bounds on the QFI, which are derived from upper and lower bounding the standard fidelity in Eq. (3).

That is, for any two functions $f_{1}\left(\rho_{\theta}, \rho_{\theta+\delta}\right)$ and $f_{2}\left(\rho_{\theta}, \rho_{\theta+\delta}\right)$ such that

$$
f_{1}\left(\rho_{\theta}, \rho_{\theta+\delta}\right) \leqslant F\left(\rho_{\theta}, \rho_{\theta+\delta}\right) \leqslant f_{2}\left(\rho_{\theta}, \rho_{\theta+\delta}\right),
$$

we can obtain induced bounds for the QFI as

$$
\mathcal{I}_{\delta}\left(f_{2} ; \rho_{\theta}\right) \leqslant I_{\delta}\left(\theta ; \rho_{\theta}\right) \leqslant \mathcal{I}_{\delta}\left(f_{1} ; \rho_{\theta}\right),
$$

where we defined the induced bound for a function $f\left(\rho_{\theta}, \rho_{\theta+\delta}\right)$ as

$$
\mathcal{I}_{\delta}\left(f ; \rho_{\theta}\right)=8 \frac{1-f\left(\rho_{\theta}, \rho_{\theta+\delta}\right)}{\delta^{2}},
$$

which in turn allows us to define bounds for the QFI in the $\delta \rightarrow 0$ limit as $\mathcal{I}\left(f ; \rho_{\theta}\right)=\lim _{\delta \rightarrow 0} \mathcal{I}_{\delta}\left(f ; \rho_{\theta}\right)$.

In what follows, we first summarize our recent result of a lower and upper bound for the QFI called the truncated QFI (TQFI) bounds [75], which are based on truncating the exact state $\rho_{\theta}$ to its largest $m$ eigenvalues [45] and computing the truncated fidelities [45]. Then, we employ the so-called suband superfidelities [76] to derive the sub-and super-quantumFisher-information (SSQFI) bounds. Here, we remark that, as discussed below, the lower TQFI bound is not just a bound on the QFI, but has additional operational meaning.

\section{Truncated QFI}

Let $\rho_{\theta}=\sum_{k=1}^{r} \lambda_{k}\left|\lambda_{k}\right\rangle\left\langle\lambda_{k}\right|$ be the spectral decomposition of the exact state, where $\lambda_{k}$ is the $k$ th eigenvalue of $\rho$ and $\left|\lambda_{k}\right\rangle$ is its associated eigenvector. Here, $1 \leqslant r \leqslant 2^{n}$ is the rank of $\rho_{\theta}$. Moreover, let us assume that the eigenvalues are ordered in decreasing order such that $\lambda_{k} \geqslant \lambda_{k+1}$. Then, for a given integer $m$ such that $1 \leqslant m \leqslant r$, we define the subnormalized states

$$
\begin{gathered}
\rho_{\theta}^{(m)}=\Pi_{\rho_{\theta}}^{m} \rho_{\theta} \Pi_{\rho_{\theta}}^{m}=\sum_{k=1}^{m} \lambda_{k}\left|\lambda_{k}\right\rangle\left\langle\lambda_{k}\right|, \\
\rho_{\theta+\delta}^{(m)}=\Pi_{\rho_{\theta}}^{m} \rho_{\theta+\delta} \Pi_{\rho_{\theta}}^{m},
\end{gathered}
$$

where $\Pi_{\rho_{\theta}}^{m}=\sum_{k=1}^{m}\left|\lambda_{k}\right\rangle\left\langle\lambda_{k}\right|$. That is, $\rho_{\theta}^{(m)}$ and $\rho_{\theta+\delta}^{(m)}$ are obtained by projecting the exact and error states, respectively, into the subspace generated by the $m$ eigenvectors of $\rho_{\theta}$ associated with its $m$ largest eigenvalues.

As shown in Refs. [45,77], the following bounds hold $\forall \delta \in$ $\mathbb{R}$

$$
F\left(\rho_{\theta}^{(m)}, \rho_{\theta+\delta}^{(m)}\right) \leqslant F\left(\rho_{\theta}, \rho_{\theta+\delta}\right) \leqslant F_{*}\left(\rho_{\theta}^{(m)}, \rho_{\theta+\delta}^{(m)}\right) .
$$

Here, $F\left(\rho_{\theta}^{(m)}, \rho_{\theta+\delta}^{(m)}\right)=\left\|\sqrt{\rho_{\theta}^{(m)}} \sqrt{\rho_{\theta+\delta}^{(m)}}\right\|_{1}$ is the truncated $f$ delity, and $F_{*}(\sigma, \tau)$ denotes the truncated generalized fidelity between two subnormalized states $\sigma$ and $\tau$, given by

$$
F_{*}(\sigma, \tau)=\|\sqrt{\sigma} \sqrt{\tau}\|_{1}+\sqrt{(1-\operatorname{Tr}[\sigma])(1-\operatorname{Tr}[\tau])} .
$$

Here, we remark that the bounds in (9) get monotonically tighter as $m$ increases, with equalities holding if $m=r$ [45]. We note that, as discussed below, the truncated fidelity bounds can be computed with $2 n+1$ qubits. Additionally, as shown in Ref. [45] the bounds in (9) are tight if (1) $\rho$ is a high-purity state or (2) $\rho$ is a low-rank state and if $m=r$.

Combining Eqs. (4)-(6) with the truncated fidelity bounds in (9) allows us to define the TQFI bounds [75]

$$
\mathcal{I}_{\delta}\left(F_{*} ; \rho_{\theta}^{(m)}\right) \leqslant I_{\delta}\left(\theta ; \rho_{\theta}\right) \leqslant \mathcal{I}_{\delta}\left(F ; \rho_{\theta}^{(m)}\right),
$$

with equalities again holding if $m=r$. We note that the quantity $\mathcal{I}\left(F_{*} ; \rho_{\theta}^{(m)}\right)$ was recently introduced in Ref. [75] and is known as the truncated quantum Fisher information (TQFI). The TQFI represents a generalization of the QFI for subnormalized states as it satisfies the canonical criteria of a QFI measure. We encourage the interested reader to see Ref. [75] for details.

\section{Sub-and superbounds}

As shown in Ref. [76], the following bounds hold $\forall \delta \in \mathbb{R}$ :

$$
\sqrt{E\left(\rho_{\theta}, \rho_{\theta+\delta}\right)} \leqslant F\left(\rho_{\theta}, \rho_{\theta+\delta}\right) \leqslant \sqrt{R\left(\rho_{\theta}, \rho_{\theta+\delta}\right)} .
$$

Here, $E(\rho, \sigma)$ and $R(\rho, \sigma)$ are called the subfidelity and superfidelity, respectively, between the quantum states $\rho$ and $\sigma$ and are defined as

$$
\begin{aligned}
& E(\rho, \sigma)=\operatorname{Tr}[\rho \sigma]+\sqrt{2\left((\operatorname{Tr}[\rho \sigma])^{2}-\operatorname{Tr}[\rho \sigma \rho \sigma]\right)} \\
& R(\rho, \sigma)=\operatorname{Tr}[\rho \sigma]+\sqrt{\left(1-\operatorname{Tr}\left[\rho^{2}\right]\right)\left(1-\operatorname{Tr}\left[\sigma^{2}\right]\right)}
\end{aligned}
$$

As shown in Appendix A 3, because these quantities are expressed as traces of products of quantum states, they can be efficiently estimated on a quantum computer requiring up to $4 n+1$ qubits $[45,76,78]$.

By combining Eqs. (4)-(6) with the sub- and superfidelity bounds in (12) we can define the sub- and super-quantumFisher-information (SSQFI) bounds

$$
\mathcal{I}_{\delta}\left(\sqrt{R} ; \rho_{\theta}^{(m)}\right) \leqslant I_{\delta}\left(\theta ; \rho_{\theta}\right) \leqslant \mathcal{I}_{\delta}\left(\sqrt{E} ; \rho_{\theta}^{(m)}\right) .
$$

It is worth noting that it has been recently shown in Ref. [79] that the sub-QFI bound is faithful to the QFI in the sense that both quantities are maximized and minimized for the same quantum states. Such a result implies that the state found by maximizing the lower bound in (15) is optimal for metrology and sensing applications. 


\section{Dynamics-agnostic QFI bounds}

One of the main advantages of the TQFI and SSQFI bounds is that that they are dynamics agnostic, meaning that their computation requires no knowledge about how the source encodes the parameter $\theta$ in $\rho$. This is in contrast to other known bounds on the QFI. For instance, for phase estimation where the state is given by $(21)$, one can show that $4\left(\operatorname{Tr}\left[\rho_{\theta}^{2} G^{2}\right]-\right.$ $\left.\operatorname{Tr}\left[\left(\rho_{\theta} G\right)^{2}\right]\right)$ is a lower bound and $4\left(\operatorname{Tr}\left[\rho_{\theta} G^{2}\right]-\left(\operatorname{Tr}\left[\rho_{\theta} G\right]\right)^{2}\right)$ is an upper bound on $I\left(\theta ; \rho_{\theta}\right)$ [69]. However, computing these bounds requires knowledge of the generator $G$, which might not always be accessible. To avoid requiring such extra knowledge, we here instead define the following dynamics-agnostic quantities from the bounds previously presented:

$$
\begin{gathered}
H_{\delta}\left(\theta ; \rho_{\theta}\right)=\max \left\{\mathcal{I}_{\delta}\left(F_{*}, \rho_{\theta}^{(m)}\right), \mathcal{I}_{\delta}\left(\sqrt{R} ; \rho_{\theta}^{(m)}\right)\right\}, \\
J_{\delta}\left(\theta ; \rho_{\theta}\right)=\min \left\{\mathcal{I}_{\delta}\left(F, \rho_{\theta}^{(m)}\right), \mathcal{I}_{\delta}\left(\sqrt{E} ; \rho_{\theta}^{(m)}\right)\right\} .
\end{gathered}
$$

Hence the following bounds on the QFI hold:

$$
H_{\delta}\left(\theta ; \rho_{\theta}\right) \leqslant I_{\delta}\left(\theta ; \rho_{\theta}\right) \leqslant J_{\delta}\left(\theta ; \rho_{\theta}\right) .
$$

It is worth noting that as shown in Ref. [45], the TQFI bounds are often tighter than the SSQFI bounds when $m \in O[\operatorname{poly}(n)]$. Hence, for large enough $m$, we will have $H_{\delta}\left(\theta ; \rho_{\theta}\right)=\mathcal{I}_{\delta}\left(F_{*}, \rho_{\theta}^{(m)}\right)$ and $J_{\delta}\left(\theta ; \rho_{\theta}\right)=\mathcal{I}_{\delta}\left(F, \rho_{\theta}^{(m)}\right)$, meaning that it will suffice to compute the TQFI bounds. Moreover, as previously mentioned, the computation of the TQFI bounds requires only $2 n+1$ qubits for $n$-qubit states $\rho$, while the computation of the SSQFI bounds (specifically the upper bound) requires $4 n+1$ qubits.

\section{VARIATIONAL QUANTUM FISHER INFORMATION ESTIMATION ALGORITHM}

In this section, we present a high-level description of the VQFIE algorithm, shown in Fig. 2. For completeness, we describe the algorithm including the optional step (dashed boxes in Fig. 2) of variationally preparing the state that maximizes the QFI for estimating the parameter $\theta$.

We remark that the VQFIE algorithm is meant to address the situation where the state of interest has a relatively high purity. Physically speaking, this would occur if one attempts to prepare a pure state on a noisy quantum device, which then results in a mixed state with high purity. Low-temperature thermal states provide another important example. Specifically, in order for the bounds in VQFIE to remain tight, the input state $\rho_{\text {in }}$ should be approximately low rank, as defined in Ref. [45]. We emphasize that such states are of significant physical interest, especially in the context of quantum sensing, where one aims to prepare a state with high purity.

We also remark that the task of estimating the QFI for such states (i.e., low-rank mixed states) is likely to be hard for classical computers. This can be seen in a number of ways. First, because the QFI can be expressed in terms of the fidelity between two quantum states, hardness results that apply to fidelity estimation (such as Ref. [45]) also apply to QFI. Second, the standard technique for QFI estimation using a classical computer involves posing the problem as a semidefinite program which has computational run time

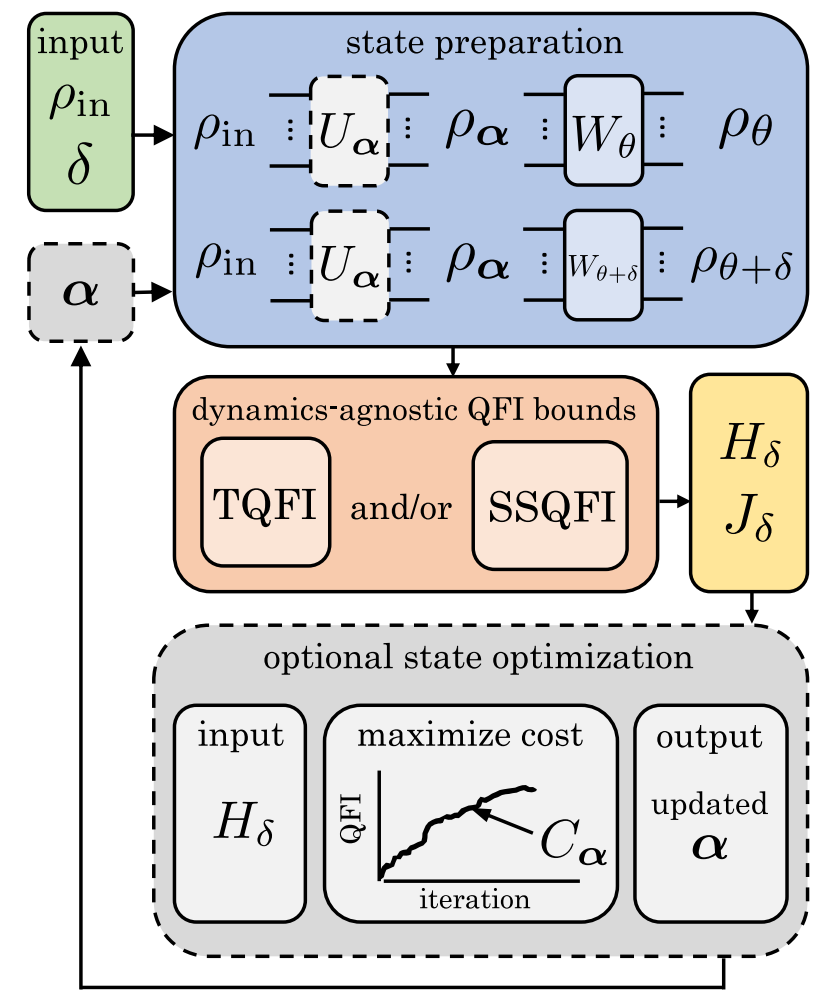

FIG. 2. Schematic diagram of the VQFIE algorithm for a unitary sensor dynamics application. VQFIE takes as input $N$ copies of an $n$-qubit state $\rho_{\text {in }}$, a value of $\delta$, and the parameters $\boldsymbol{\alpha}$ of a parametrized unitary $U_{\alpha}$. The computation of the TQFI bound requires $N=2$, while that of the SSQFI requires $N=4$. After the (optional) application of $U_{\alpha}$, half of the probe states evolve under the action of a unitary $W_{\theta}=e^{-i \theta G}$ as in (21), while the other half evolve under the action of $W_{\theta+\delta}$, resulting in $N / 2$ exact states $\rho_{\theta}$ and $N / 2$ error states $\rho_{\theta+\delta}$. Then, VQFIE computes the dynamics-agnostic bounds $H_{\delta}$ and $J_{\delta}$ of Eqs. (16) and (17). The lower bound $H_{\delta}$ on the QFI can be employed as a cost function in a quantum-classical hybrid optimization loop to train the parameters $\boldsymbol{\alpha}$ and variationally prepare the state that maximizes the QFI

polynomial in the dimension of the states [74]. Because this dimension scales exponentially with the number of qubits, such techniques are not scalable to problem sizes of interest. As such, a quantum computer could provide an advantage in the task of estimating the QFI of an arbitrary mixed quantum state.

\section{A. Algorithm structure}

\section{State preparation}

As schematically depicted in Fig. 2, the input to the VQFIE algorithm consists of $N$ copies of an $n$-qubit input state $\rho_{\text {in }}$, a value of $\delta$, and a set of parameters $\boldsymbol{\alpha}$ which parametrize a unitary $U_{\boldsymbol{\alpha}}$. When computing the TQFI bounds, we have $N=$ 2 , whereas the computation of the SSQFI bounds requires $N=4$ copies of $\rho_{\text {in. }}$. In both cases, we can first apply the parametrized unitary to the input state to obtain a variational probe state

$$
\rho_{\alpha}=U_{\alpha} \rho_{\text {in }} U_{\alpha}^{\dagger}
$$


The goal of $U_{\alpha}$ is to map the input state $\rho_{\text {in }}$ to the state that maximizes the QFI. Note that if one just wants to estimate the QFI, and not variationally maximize it, one need not apply $U_{\boldsymbol{\alpha}}$ to the input state.

Since no knowledge about the dynamics of the source is assumed, we employ a hardware-efficient Ansatz [80] for the parametrized unitary $U_{\alpha}$. This Ansatz reduces the circuit depth overhead when implementing VQFIE on a quantum computer by expressing $U_{\alpha}$ as a sequence of gates taken from an alphabet of native gates to the specific hardware employed. Hence, without loss of generality, we can write

$$
U_{\alpha}=\prod_{\mu} e^{-i \alpha_{\mu} V_{\mu}} \Gamma_{\mu},
$$

where $\Gamma_{\mu}$ are unparametrized unitaries and $V_{\mu}$ are Hermitian operators. For the numerical implementations in this paper we employ a layered hardware-efficient Ansatz, where gates are arranged in a bricklike structure acting on alternating pairs of neighboring qubits [56]. Such an architecture can be readily implemented in a quantum computer with local qubit connectivity.

After the optional action of $U_{\alpha}$, half of the states interact with a source that encodes the information of a parameter $\theta$ as

$$
\rho_{\theta}=W_{\theta} \rho W_{\theta}^{\dagger} \quad \text { with } W_{\theta}=e^{-i \theta G},
$$

while the source encodes the information of $\theta+\delta$ in the remaining half. That is, we obtain $N / 2$ exact states $\rho_{\theta}$ and $N / 2$ error states $\rho_{\theta+\delta}$. Note that, as shown in Fig. 2, our algorithm does not require knowledge of the generator of the interaction between the quantum state and the source, that is, we do not require knowledge about what the Hermitian operator $G$ is. At this stage, it is important to distinguish between $\boldsymbol{\alpha}$ and $\theta$. The former is a set of parameters which can be variationally updated to prepare the near-optimal state for sensing applications, while the latter is the parameter that one is attempting to estimate (e.g., $\theta$ is the magnetic field amplitude $B$ in the case of magnetometry).

\section{Computation of bounds}

The next step in VQFIE is the computation of the QFI bounds. As indicated in (16) and (17), one needs to compute the TQFI and the SSQFI bounds. Here, we briefly describe how each one of those quantities can be estimated on a quantum computer.

The SSQFI bounds are obtained by computing the suband superfidelities of Eqs. (13) and (14). The terms in $E\left(\rho_{\theta}, \rho_{\theta+\delta}\right)$ and $R\left(\rho_{\theta}, \rho_{\theta+\delta}\right)$ of the form $\operatorname{Tr}\left[\rho_{\theta}^{2}\right]$ or $\operatorname{Tr}\left[\rho_{\theta} \rho_{\theta+\delta}\right]$ can be computed with $2 n$ qubits by means of the destructive SWAP test [81]. The destructive SWAP test employs a constant-depth quantum circuit with classical postprocessing that scales linearly in the number of qubits [81]. For the $\operatorname{Tr}\left[\rho_{\theta} \rho_{\theta+\delta} \rho_{\theta} \rho_{\theta+\delta}\right]$ term, one can employ a generalized SWAP test (e.g., Refs. [78,82]) involving a controlled permutation gate, whose circuit depth scales linearly in the number of qubits. For completeness, we show how the generalized SWAP test can be used to estimate these functionals in Appendix A 3. As shown there, these circuits are efficient in the problem size. Finally, we remark that one can also compute the SSQFI bounds via the circuits introduced in Ref. [76].
Computing the TQFI bounds is a more involved procedure and requires a variational subroutine. Specifically, we will need to obtain the $m$ largest eigenvalues and associated eigenvectors of $\rho_{\theta}$. These are obtained using the variational quantum state eigensolver algorithm [48], which variationally diagonalizes the state $\rho_{\theta}$ over the subspace of its $m$ principal components. Specifically, one trains a parametrized gate sequence to achieve this subspace diagonalization task. The subroutine then returns estimates of the $m$ largest eigenvalues and their associated eigenvectors, denoted $\left\{\tilde{\lambda}_{i}\right\}_{i=1}^{m}$ and $\left\{\left|\tilde{\lambda}_{i}\right\rangle\right\}_{i=1}^{m}$, respectively. We refer the reader to Appendix A for additional details of these subroutines.

After this variational subroutine, one then runs several nonvariational quantum circuits to compute the overlap between $\rho_{\theta+\delta}$ and the estimates of the principal components of $\rho_{\theta}$, i.e., the states in the set $\left\{\left|\tilde{\lambda}_{i}\right\rangle\right\}_{i=1}^{m}$. These overlaps are then combined with classical postprocessing as described in Ref. [45], in order to compute the upper and lower bounds on the fidelity appearing in (9). We remark that Ref. [45] showed that this procedure scales efficiently with problem size. Here, we remark that the efficiency in estimating the TQFI bounds relies on the efficiency of the variational diagonalization subroutine [48].

\section{Classical parameter update}

When preparing the optimal probe state $\rho_{\boldsymbol{\alpha}}$, the final step of each VQFIE iteration is a classical parameter update. Here, our algorithm learns the parameters $\boldsymbol{\alpha}$ that approximately maximize the cost function

$$
C_{\alpha}=H_{\delta}\left(\theta ; \rho_{\theta}\right),
$$

where we note that the dependence on $\boldsymbol{\alpha}$ that arises from the preparation unitary is left implicit to simplify the notation. Here, a hybrid quantum-classical optimizer employs the value of the cost (or its gradient) to update the preparation parameters $\boldsymbol{\alpha}$. The whole algorithm then repeats until stopping criteria are met. The probe state from the final iteration, which approximately maximizes Eq. (22), is then used to calculate the upper bounds. The estimation of the QFI is then between $H_{\delta}$ and $J_{\delta}$.

\section{B. Gradient scaling}

Significant progress has recently been made on studying the scaling of gradients in VQAs [55-60]. This includes identifying some conditions under which the gradient vanishes exponentially in $n$, known as a barren plateau landscape.

We now proceed to argue that VQFIE does not exhibit a barren plateau landscape when a shallow-depth Ansatz is employed. In particular, here we will use the results in Ref. [56] that connect the existence of barren plateaus in VQAs to the locality of the cost function. Specifically, it was shown that global cost functions, i.e., cost functions where one computes the expectation value of operators acting nontrivially on all qubits, lead to barren plateaus. On the other hand, local cost functions, i.e., cost functions where one computes the expectation value of operators acting nontrivially on a small subset of qubits, do not exhibit barren plateaus for shallow Ansätze [56]. Hence we aim to argue that the cost functions employed in VQFIE are local, rather than global, in nature. 
First, we consider the variational subroutine used to compute the TQFI bounds. This is the variational quantum state eigensolver, proposed in Ref. [48], and therein a local cost function was proposed for the diagonalization task of interest. We refer the reader to Ref. [48] for details of this local cost function. However, it suffices to say that the cost landscape would not have a barren plateau so long as the Ansatz depth is sufficiently shallow, i.e., $O\left[\log _{2}(n)\right]$ depth [56].

Second, we consider the variational optimization of the $\boldsymbol{\alpha}$ parameters in the state preparation unitary. This involves the cost function in (22), which takes the maximum between the lower bounds provided by the TQFI and the SSQFI. In our implementation of VQFIE, we found the TQFI to typically provide a tighter bound [45], so we focus our discussion here on the TQFI. From Ref. [75], we can write the TQFI lower bound in the limit $\delta \rightarrow 0$ as

$$
\begin{aligned}
\mathcal{I}\left(F_{*} ; \rho_{\theta}^{(m)}\right)= & 4 \operatorname{Tr}\left[\rho_{\theta}^{(m)} G^{2}\right]-\sum_{i, j=1}^{m} \frac{8 \lambda_{i} \lambda_{j}}{\lambda_{i}+\lambda_{j}}\left|G_{i j}\right|^{2} \\
& -4 \operatorname{Tr}\left[\Pi_{\rho_{\theta}}^{\bar{m}} G \rho_{\theta}^{(m)} G\right],
\end{aligned}
$$

with $G_{i j}=\left\langle\lambda_{i}|G| \lambda_{j}\right\rangle$, and where $\Pi_{\rho_{\theta}}^{m}+\Pi_{\rho_{\theta}}^{\bar{m}}=\mathbb{1}$.

In this form, we see that the first term is a local cost function [56], so long as $G$ is local, and hence its gradient should remain large for a shallow-depth Ansatz. For instance, for a magnetometry application (see below), one has $G=\sum_{i=1}^{n} Z_{i}$, and hence $G^{2}=n \mathbb{1}+\sum_{i \neq j} Z_{i} Z_{j}$ contains terms that act nontrivially on at most two qubits. In contrast, the last term is a global cost function. Recalling that $\Pi_{\rho_{\theta}}^{\bar{m}}=\sum_{k=m+1}^{d}\left|\lambda_{k}\right\rangle\left\langle\lambda_{k}\right|$, the final term in (23) is of the form $\sum_{k=m+1}^{d} \operatorname{Tr}\left[G\left|\lambda_{k}\right\rangle\left\langle\lambda_{k}\right| G \rho_{\theta}^{(m)}\right]$, and one computes the expectation value of the global operators $G\left|\lambda_{k}\right\rangle\left\langle\lambda_{k}\right| G$. (Note that this term is global regardless of the locality of $G$.) Hence the contribution to the cost function gradient associated with this final term will be exponentially suppressed. Finally, we note that the middle term is likely to have a smaller gradient than the first term even though it is not a fully global term. Hence we expect that the first term in this expression will have the largest gradient magnitude, and as a result the overall gradient magnitude will not vanish exponentially for an $O\left[\log _{2}(n)\right]-$ depth Ansatz.

To further support the claims that our algorithm has a local cost function, and hence does not exhibit a barren plateau for shallow Ansätze, we refer the reader to Sec. IV. Therein we numerically analyze the trainability of our cost function.

\section{NUMERICAL SIMULATION}

In this section, we present our numerical results obtained from simulating the VQFIE algorithm. Specifically, we train the parameters in $U_{\alpha}$ in order to prepare the probe state that maximizes the QFI for a magnetometry application. Hence we consider a system of $n$ spin-1/2 particles ( $n$ qubits) interacting with a uniform magnetic field. The Hamiltonian is modeled as

$$
G=\sum_{i=1}^{n} Z_{i}
$$

with $Z_{i}$ being the Pauli $z$ operator on qubit $i$. The parameter $\theta$ appearing in Eq. (21) is the phase acquired by spins, after precessing for some time under the action of the magnetic field.

Here, we recall that if the probe state is pure $\rho_{\alpha}=$ $\left|\psi_{\alpha}\right\rangle\left\langle\psi_{\alpha}\right|$, then it is well known that the optimal probe state corresponds to the $\mathrm{GHz}$ state $|\mathrm{GHz}\rangle=\left(|0\rangle^{\otimes n}+e^{i \varphi}|1\rangle^{\otimes n}\right) / \sqrt{2}$ with $\varphi \in \mathbb{R}$, and the QFI reaches the Heisenberg limit

$$
\max _{\left|\psi_{\alpha}\right\rangle} I\left(\theta,\left|\psi_{\theta}\right\rangle\left\langle\psi_{\theta}\right|\right)=4 n^{2} .
$$

Here, $\left|\psi_{\theta}\right\rangle=W_{\theta}\left|\psi_{\alpha}\right\rangle$. Moreover, if the probe state $\rho_{\alpha}$ is mixed, then the optimal state can be obtained from Ref. [36], and its associated QFI is

$$
\max _{\rho_{\boldsymbol{\alpha}}} I\left(\theta, \rho_{\theta}\right)=\frac{1}{2} \sum_{k=1}^{d} \lambda_{k, d-k+1}\left(g_{k}-g_{d-k+1}\right)^{2},
$$

where $g_{k}$ are the eigenvalues of $G$ ordered in decreasing order and where $d=2^{n}$. Here, $\lambda_{k, l}=0$ if $\lambda_{k}=\lambda_{l}=0$ and $\lambda_{k, l}=\left(\lambda_{k}-\lambda_{l}\right)^{2} /\left(\lambda_{k}+\lambda_{l}\right)$ otherwise [36], and we recall that $\lambda_{k}$ is the $k$ th eigenvalue of the probe state $\rho_{\boldsymbol{\alpha}}$. Note that in order to compute (26), one must have perfect knowledge of all the eigenvalues of $\rho_{\boldsymbol{\alpha}}$ and of the generator $G$. Hence such a quantity is not efficiently computable in practice. However, here we employ Eqs. (25) and (26) to benchmark the maximum QFI obtained by training the parameters in $U_{\boldsymbol{\alpha}}$.

\section{A. Performance of VQFIE in experimentally relevant regimes}

For our heuristics we simulated the VQFIE algorithm without sampling noise. Moreover, the cost function optimization was performed by employing the constrained optimization by linear approximation (COBYLA) algorithm [83]. For each case analyzed we ran 30 instances of VQFIE, each with 200 cost optimization iterations, and we present the results of the run that achieved the largest final cost function value. We remark that for $U_{\boldsymbol{\alpha}}$ we employed a layered hardware-efficient Ansatz with three layers composed of single-qubit rotations and CNOT gates.

In Fig. 3(a) we show results for an $n=4$ qubit implementation of the VQFIE algorithm for a randomly generated mixed state with a purity of 0.95 and for different values of $m$ in the truncated state $\rho_{\theta}^{(m)}$. In all cases, the TQFI lower bound was tighter than the SSQFI lower bound so that $H_{\delta}\left(\theta ; \rho_{\theta}\right)=$ $\mathcal{I}_{\delta}\left(F_{*}, \rho_{\theta}^{(m)}\right)$. Here, we can verify that the TQFI lower bound becomes tighter with increasing $m$. Note that the improvement in the $m=4$ case is not as significant due to the fact that smaller eigenvalues give rise to smaller improvements. This is due to the fact that most of the information in the state is encoded in the subspace spanned by the eigenvectors associated with the largest eigenvalues [45].

Let us now analyze the performance of VQFIE for different purities. In Fig. 3(b) we present results for randomly generated $n=4$ input states with purities of $0.75,0.8, \ldots, 0.95$. Let us first remark that the maximum QFI achievable increases with the purity, as shown by the vertical lines obtained from Eq. (26). In all cases we chose $m=4$, and for all purities we found that the TQFI lower bound is tighter than the SSQFI lower bound. Moreover, we can also see that, as expected, VQFIE has a better performance for high-purity states, as the final cost value is larger for higher purities. This again can be explained from the fact that in low-purity states, more 
(a)
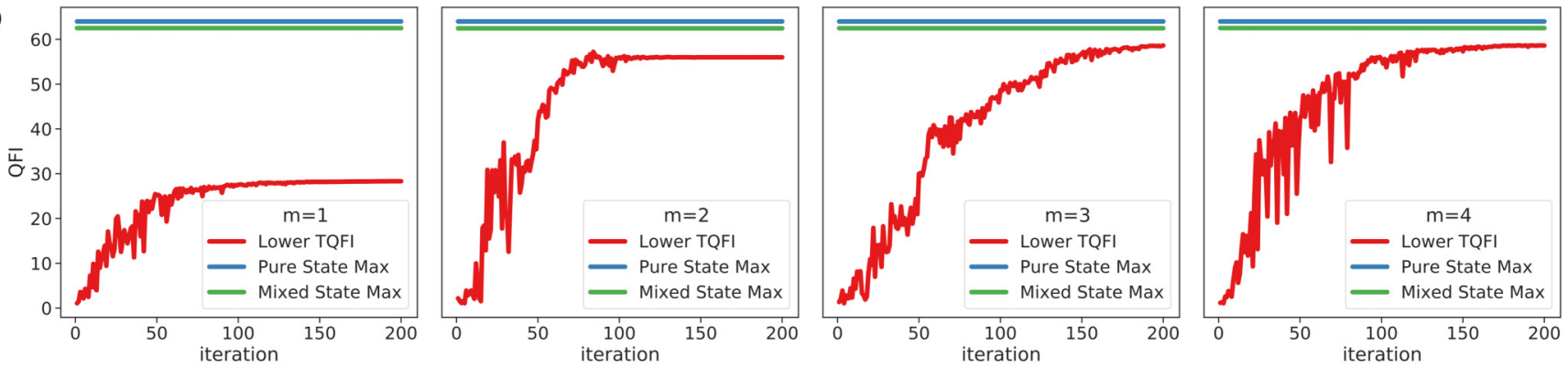

(b)
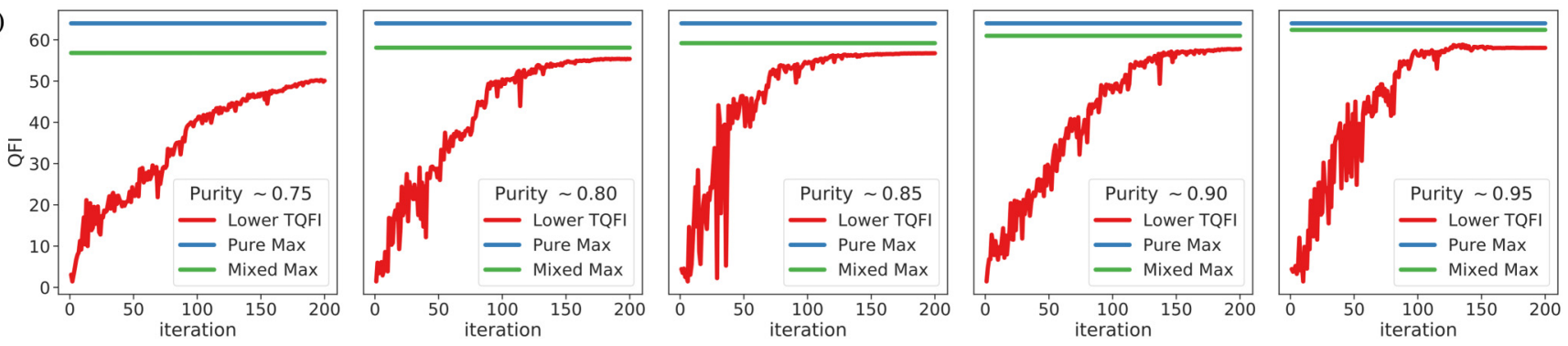

FIG. 3. Cost function value vs iteration for an $n=4$ implementation of VQFIE. (a) Panels correspond to a different value of $m$ for the TQFI lower bound. The input state was randomly generated with a purity of 0.95 , and the error state was created with $\delta=0.1$. In all cases the TQFI is tighter than the SSQFI. The plots show that the performance of VQFIE improves as $m$ increases since VQFIE reaches higher cost values. (b) Panels correspond to randomly generated states with different purities. In all cases the error state was created with $\delta=0.1$. We see that VQFIE reaches higher cost values for higher-purity states. Horizontal lines depict the maximal QFI for pure states [Eq. (25)] and for mixed states [Eq. (26)]

information is encoded in a larger number of eigenvalues and the subspace generated by their associated eigenvectors. Hence truncating the state to $m=4$ leads to information loss and to looser truncated bounds. Similarly, the SSQFI bounds are also loose for low purities due to the looseness of the suband superfidelities in this case [45].

We note that, for quantum metrology applications, one wishes to prepare probe states that are typically low rank (high purity), as states that are close to being maximally mixed are not good candidates for quantum sensors [36]. Hence it is fortunate that our algorithm has a better performance in this high-purity regime.

\section{B. Scaling of the cost function landscape for VQFIE}

In this section we numerically analyze the presence or absence of barren plateaus in the VQFIE cost function landscape. Specifically, we consider here the case when one trains the parameters $\boldsymbol{\alpha}$ via the TQFI lower bound. In Sec. III B, we argued that in this case the cost function is local and hence that no barren plateau arises for shallow hardware-efficient Ansätze (i.e., with $O\left[\log _{2}(n)\right]$ depth). Here, we recall that the standard way to numerically analyze the existence of barren plateaus is by computing the variance of the cost function partial derivative, leading to the following definition.

Definition 1. A cost function $C_{\alpha}$ exhibits a barren plateau if $E_{\alpha}\left[\partial_{\mu} C_{\alpha} / n^{2}\right]=0$ and if

$$
\operatorname{Var}_{\alpha}\left[\partial_{\mu} C_{\alpha} / n^{2}\right] \in O\left(1 / 2^{n}\right),
$$

where $\partial_{\mu} C_{\boldsymbol{\alpha}} \equiv \partial C_{\boldsymbol{\alpha}} / \partial \alpha_{\mu}$ for some $\alpha_{\mu} \in \boldsymbol{\alpha}$.

Here, the expectation values are taken over the parameters $\boldsymbol{\alpha}$, and we divided the cost function by the normalization factor $n^{2}$. Note that (27) implies that the cost function gradients are (on average) exponentially suppressed across the landscape and hence that the landscape is essentially flat for large problem sizes.

Recently, it was shown in Ref. [84] that the presence of barren plateaus can also be diagnosed via the variance of difference in cost function values, i.e., by analyzing the scaling of $\operatorname{Var}_{\boldsymbol{\alpha}, \boldsymbol{\alpha}^{\prime}}\left[\Delta C_{\boldsymbol{\alpha}, \boldsymbol{\alpha}^{\prime}} / n^{2}\right]$, where $\Delta C=C_{\boldsymbol{\alpha}}-C_{\boldsymbol{\alpha}^{\prime}}$. The main advantage here is that $\operatorname{Var}_{\boldsymbol{\alpha}, \boldsymbol{\alpha}^{\prime}}\left[\Delta C_{\boldsymbol{\alpha}, \boldsymbol{\alpha}^{\prime}} / n^{2}\right]$ is computationally cheaper to compute, as it requires fewer quantum circuit evaluations [84]. Hence we have the following alternative definition.

Definition 2. A cost function $C_{\alpha}$ exhibits a barren plateau if $E_{\alpha}\left[\partial_{\mu} C_{\alpha} / n^{2}\right]=0$ and if

$$
\operatorname{Var}_{\boldsymbol{\alpha}, \boldsymbol{\alpha}^{\prime}}\left[\Delta C_{\boldsymbol{\alpha}, \boldsymbol{\alpha}^{\prime}} / n^{2}\right] \in O\left(1 / 2^{n}\right) .
$$

In Fig. 4, we show numerical results for $\operatorname{Var}_{\boldsymbol{\alpha}, \boldsymbol{\alpha}^{\prime}}\left[\Delta C_{\boldsymbol{\alpha}, \boldsymbol{\alpha}^{\prime}} / n^{2}\right]$ versus the number of qubits $(n=2,3, \ldots, 13)$ for the VQFIE cost function defined in Eq. (22). We employ a hardware-efficient Ansatz with $\log _{2}(n)$ layers for the magnetometry application of Eq. (24), with different values of $\delta=$ $0.1,0.5,1$. For $n=2, \ldots, 11$ we computed by variance by averaging over 1000 random parameter initializations, while for $n=12,13$ we averaged over 100 initializations. Here, we can we see that for $\delta=1$ the variance of cost function differences vanishes exponentially with the system size (as noted by a straight line in the log-linear plot). In this case, the cost function is clearly global as one compares two states in an exponentially large Hilbert space whose fidelity is (on average) exponentially small. Such a case is similar to that analyzed in Ref. [56], where a cost function defined as the overlap between two quantum states that are not necessarily close exhibits a barren plateau.

On the other hand, as $\delta$ decreases, the scaling of $\operatorname{Var}_{\boldsymbol{\alpha}, \boldsymbol{\alpha}^{\prime}}\left[\Delta C_{\boldsymbol{\alpha}, \boldsymbol{\alpha}^{\prime}} / n^{2}\right]$ drastically improves since the cost 


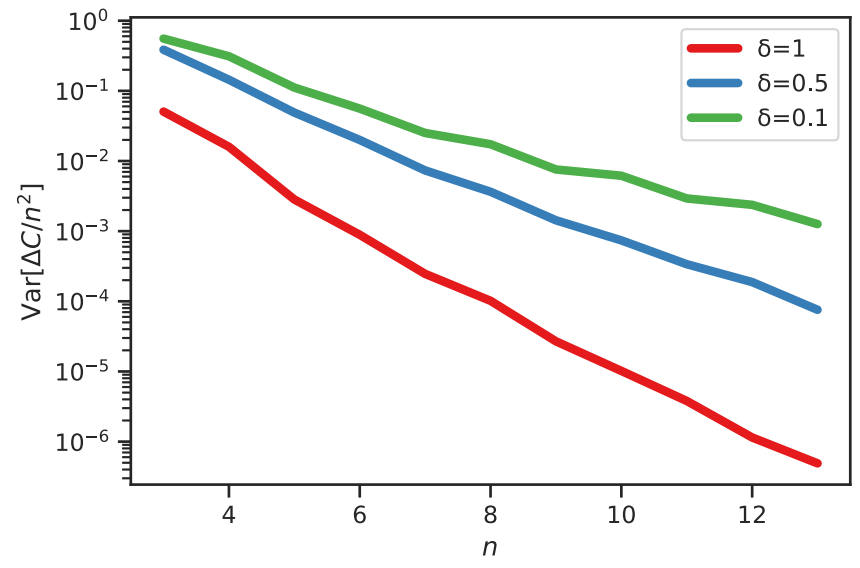

FIG. 4. Normalized variance of the VQFIE cost function difference vs number of qubits. As shown in Ref. [84], the $y$ axis can be viewed as a proxy for the trainability of the cost function. If the variance of the cost difference vanishes exponentially with the number of qubits, the cost exhibits a barren plateau. However, for $\delta=0.1$ we see that this variance does not decrease exponentially with problem size. Namely, since the $y$ axis is presented in a $\log$ scale and since the green curve deviates significantly from a straight line, the scaling is nonexponential.

becomes local and Eq. (23) holds. Specifically, for $\delta=0.1$ the variance $\operatorname{Var}_{\boldsymbol{\alpha}, \boldsymbol{\alpha}^{\prime}}\left[\Delta C_{\boldsymbol{\alpha}, \boldsymbol{\alpha}^{\prime}} / n^{2}\right]$ clearly does not vanish exponentially with $n$, which means that the cost function does not exhibit a barren plateau. This nonexponential scaling is characteristic of local cost functions [56]. Moreover, one can see that $\operatorname{Var}_{\boldsymbol{\alpha}, \boldsymbol{\alpha}^{\prime}}\left[\Delta C_{\boldsymbol{\alpha}, \boldsymbol{\alpha}^{\prime}} / n^{2}\right]$ is increased by orders of magnitude by reducing the value of $\delta$. Taken together, these results show that in the small-delta limit, the cost function trainability is improved as the cost function becomes local.

\section{COMPARISON WITH LITERATURE}

In this section we heuristically compare the TQFI and the SSQFI lower bounds with the so-called purity-loss bound, a dynamics-agnostic lower bound for the QFI [69,70]. As shown below, the bounds presented in this paper are tighter than the purity-loss bound for all cases considered.

As shown in Refs. [69,70], the following lower bound on the QFI holds:

$$
\mathcal{L}\left(\theta ; \rho_{\theta}\right) \leqslant \mathcal{I}\left(F_{*} ; \rho_{\theta}^{\left(m^{*}\right)}\right),
$$

where $\mathcal{L}\left(\theta ; \rho_{\theta}\right)=4\left(\operatorname{Tr}\left[\rho^{2} G^{2}\right]-\operatorname{Tr}[\rho G \rho G]\right)$. However, this quantity can also be expressed as [69]

$$
\mathcal{L}\left(\theta ; \rho_{\theta}\right) \approx 2 \frac{\Delta v}{(\Delta x)^{2}},
$$

where $\Delta v$ is the purity loss given by

$$
\Delta v=\operatorname{Tr}\left[\rho^{2}\right]-\operatorname{Tr}\left[\rho_{\text {ave }}^{2}\right] .
$$

Here, $\rho_{\text {ave }}$ is the the ensemble-averaged state obtained by considering that the parameter $\theta$ is not stable during an experiment but rather is subject to statistical fluctuations. Hence each time the probe state interacts with the source, the unitary $W_{\theta(x)}$ is applied with some probability $p_{x}$. Finally, $\theta(x)$ is a random variable normally distributed about $\theta$ with some variance $(\Delta x)^{2} \ll 1$, and we define $\rho_{\text {ave }}=\sum_{x} p_{x} \rho_{\theta(x)}$ [69]. Hence $\mathcal{L}\left(\theta ; \rho_{\theta}\right)$ quantifies how fragile the probe state is to stochastic fluctuations in the parameter $\theta$.

In Fig. 5 we compare the TQFI and the SSQFI bounds with the purity-loss bound of (30) for different system sizes ( $n=4,6,8)$ and for states with purities in the range $(1 / n, 1)$. In all cases, the probe state was the mixed state that leads to the maximized QFI of (26) from Ref. [36]. Just as with the shift parameter $\delta$ in Eq. (3), one would ideally minimize $(\Delta x)^{2}$; however, achievable ranges depend on experimental capabilities. So, to fairly compare with Ref. [70], we set $\delta=(\Delta x)^{2}=0.1$, as this was the value used in their experiment. Here, we remark that the TQFI bounds were computed with $m=4$, while the purity of the average state $\rho_{\text {ave }}$ was obtained by stratified sampling from a discretized Gaussian distribution with $K$ samples (or strata) [70]. We refer the reader to Appendix B for a detailed discussion of how the number of strata was obtained for a fair comparison between bounds.

As shown in Fig. 5, the VQFIE lower bound of (16) is tighter than the purity-loss bound for all values of $n$ and purities considered. In fact, both the TQFI and the SSQFI lower bounds are individually tighter than the purity-loss bound, with the only exception being $n=4$. Here, $\mathcal{L}\left(\theta ; \rho_{\theta}\right)$ is larger than the TQFI lower bound for purities smaller than $1 / 2$. As previously mentioned, this is expected due to the fact that the TQFI bounds are loose for low-purity states. Moreover, we can also see that for $n=6$ and $n=8$ the VQFIE bounds are noticeably better than the purity-loss bounds. This can be due to the fact that for (30) to hold, one requires $(\Delta x)^{2} \ll 1$, which is not always the case [70].

Finally, let us remark that Fig. 5 also shows that the VQFIE upper and lower bounds can be very tight for high purities. This means that $J_{\delta}-H_{\delta}$ will give a small interval where the QFI actually lies, and hence VQFIE outputs a precise estimate of the QFI in this purity range.

\section{CONCLUSION}

In this paper, we presented an algorithm designed for NISQ devices to estimate the quantum Fisher information (QFI), called variational quantum Fisher information estimation (VQFIE). For this purpose, we introduced upper and lower bounds on the QFI that are based on bounding the fidelity. These bounds are then efficiently computed on a quantum computer, as part of our proposed algorithm.

Specifically, we presented two types of bounds on the QFI that are conceptually distinct, in that while both attempt to tackle the problem of computing the quantum fidelity (a nonlinear function of quantum states), the approach used is different in each case. One of our bounds is based on truncating the spectrum of the exact state and reducing the complexity of computing the nonlinear function on (subnormalized) quantum states. These are called the truncated quantum Fisher information (TQFI) bounds. Our other bounds are based on bounds for the quantum fidelity known as the sub- and superfidelity, which replace the nonlinear function in the fidelity by linear or quadratic functions. In this case, the bounds are called the sub- and super-quantum-Fisherinformation (SSQFI) bounds. 

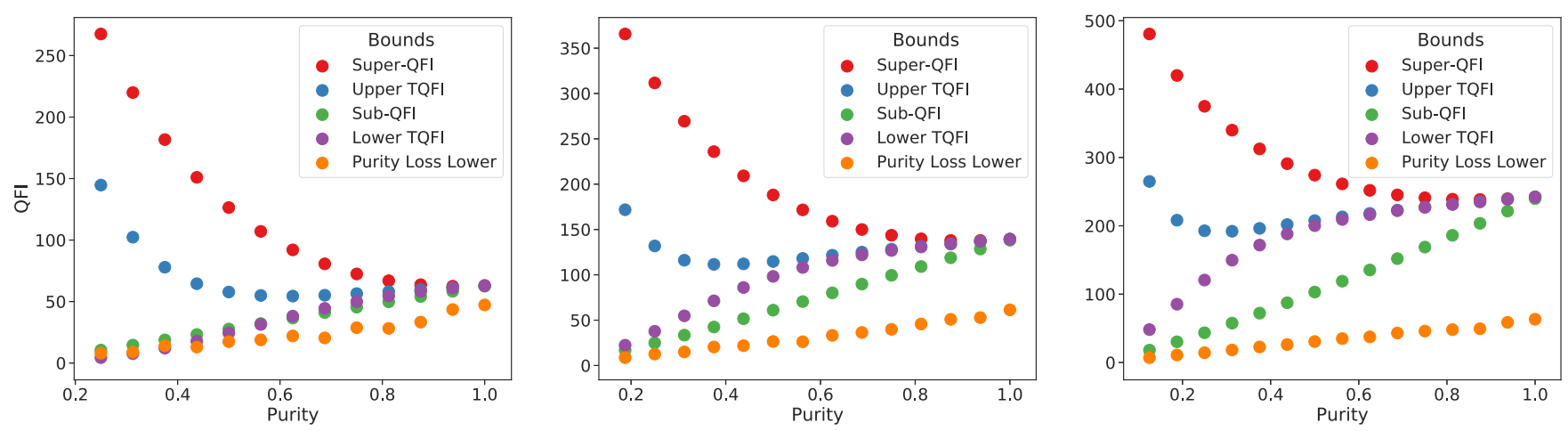

FIG. 5. VQFIE and purity-loss bounds. Here, we present the TQFI, the SSQFI, and the purity-loss bounds on the QFI for different system sizes $(n=4,6,8)$ and for purities in $(1 / n, 1)$. For each value of the purity the bounds were evaluated at the optimal probe state of Ref. [36], which maximizes the mixed-state QFI in Eq. (26). Here, we can see that in all cases considered the VQFIE lower bounds of (16) are tighter than the purity-loss bound in (30). Moreover, the plots also show that the VQFIE bounds in Eq. (18) are tighter for high purities, i.e., in the range of purities for which VQFIE is aimed.

We especially focused on the TQFI bounds. Our previous work established the TQFI lower bound as being operationally meaningful [75]. The present paper focuses on computing the TQFI bounds using a variational quantum algorithm, and we show how the maximization of the lower bound over state preparations can be used to enhance quantum sensing capabilities.

Although we found the TQFI to be tighter for the magnetometry Hamiltonian considered, in general this may not be the case. As such, we include the possibility in our algorithm to include both TQFI and SSQFI bounds, keeping only the tightest in the end. Although the TQFI bounds were tighter in our specific implementation, there were two distinct benefits to investigating the SSQFI bounds. First, in a companion paper [79], we proved that the sub-QFI is a faithful lower bound, meaning that the state that maximizes the sub-QFI is the same state that maximizes the QFI. Second, the SSQFI bounds do not require the variational quantum subroutine to truncate the state to the principal components.

In addition to introducing the general algorithm, we provided qualitative and quantitative arguments as to why our algorithm avoids barren plateaus for shallow-depth Ansätze and for small $\delta$ values, suggesting a favorable scaling for the gradient magnitude and hence a favorable scaling for training.

While trainability is a crucial consideration when proposing lower bounds for use in VQA cost functions, so too is the tightness of the proposed bounds. As illustrated in our numerical results, we expect our bounds to be tighter as the purity of the state increases. This is also true for other bounds on the QFI in the literature. For the magnetometry example that we considered, we found that our bounds were tighter than recently proposed literature bounds, over a range of different purity values.

In addition to tightness and trainability, another key aspect of VQFIE is the fact that our bounds are agnostic to the underlying dynamics. Computing our bounds does not require knowledge of the generator of the dynamics. This is useful for quantum sensing tasks for systems that are either complicated or not fully characterized. Hence this makes the VQFIE algorithm broadly applicable.
VQFIE is a promising algorithm for implementation in the NISQ era. Because the quantum Cramér-Rao bound (QCRB) is ubiquitously used as a figure of merit in experiments, efficiently and accurately estimating the QFI (upon which the QCRB directly depends) is a crucially important task in quantum sensing. As such, we expect that our algorithm will find broad applicability in evaluating the performance of quantum sensors in the fields of chemistry, biology, materials science, and cosmology.

\section{ACKNOWLEDGMENTS}

We are grateful to Tyler Volkoff, Jing Liu, and Haidong Yuan for helpful discussions. This work was supported by the Quantum Science Center (QSC), a National Quantum Information Science Research Center of the U.S. Department of Energy (DOE). J.L.B. was initially supported by the U.S. DOE through a quantum computing program sponsored by the Los Alamos National Laboratory (LANL) Information Science \& Technology Institute. J.L.B. was also supported by a National Science Foundation Graduate Research Fellowship under Grant No. 1650115. M.C. and A.S. also acknowledge initial support from the Center for Nonlinear Studies at LANL. A.S. is now supported by the internal R\&D from Aliro Technologies, Inc. P.J.C. also acknowledges initial support from the LANL ASC Beyond Moore's Law project.

\section{APPENDIX A: DETAILS OF THE VQFIE ALGORITHM}

This Appendix aims to make our paper more self-contained by providing additional details of the different VQFIE subroutines employed to compute the TQFI bounds. However, we also refer the reader to the original papers on state diagonalization [48] and fidelity estimation [45].

\section{State diagonalization}

Let us here describe the variational quantum state eigensolver (VQSE) algorithm of Ref. [48]. As described in the main text, the VQSE is employed to obtain approximations of the $m$ largest eigenvalues of a state and to prepare their associated approximated eigenvectors. Here, we recall that we 
use this algorithm as a subroutine to compute the TQFI as schematically shown in Fig. 2.

The VQSE algorithm takes in as input an integer $m$, a quantum state $\rho$, and a set of parameters $\boldsymbol{\beta}$ used to parametrize a diagonalizing gate sequence which we denote as $V_{\beta}$. Then, the output consists of estimates of the $m$ largest eigenvalues and their associated eigenvectors. Here, the algorithm aims to minimize a cost function of the form

$$
C_{\beta}=\operatorname{Tr}\left[H V_{\beta} \rho V_{\beta}^{\dagger}\right],
$$

for some Hamiltonian $H$ diagonal in the computational basis such that its $m$ lowest eigenenergies are nondegenerate. This cost function exploits the close connection between majorization and diagonalization, as the cost is minimized if $V_{\beta}$ maps the $k$ th-largest eigenvector of $\rho$ to the $k$ th-smallest energy eigenstate of $H$.

The parameters in $\boldsymbol{\beta}$ are trained in a hybrid quantumclassical optimization loop whose trainability is guaranteed from the fact that one can always choose $H$ to be a local Hamiltonian [56]. Once the optimal parameters $\boldsymbol{\beta}$ have been obtained, it is then straightforward to extract approximations of the largest eigenvalues $\left\{\widetilde{\lambda}_{i}\right\}_{i=1}^{m}$ and their associated eigenvectors $\left\{\left|\widetilde{\lambda}_{i}\right\rangle\right\}_{i=1}^{m}$. To estimate the $m$ largest eigenvalues, one simply performs the optimal gate sequence, $V_{\beta}$, on $\rho_{\theta}$ and then measures in the computational basis. Mathematically, we have

$$
\tilde{\lambda}_{i}=\left\langle z_{i}\left|V_{\boldsymbol{\beta}_{\mathrm{opt}}} \rho_{\theta} V_{\boldsymbol{\beta}_{\mathrm{opt}}}^{\dagger}\right| z_{i}\right\rangle,
$$

and in practice, one simply measures the approximately diagonalized state a finite number of times. We denote this number as $N_{\text {runs }}$. Then, for a bit string $z_{i}$ with frequency of occurrence $f_{i}$, the eigenvalues are estimated as

$$
\tilde{\lambda}_{i} \approx \frac{f_{i}}{N_{\text {runs }}}
$$

Once the $m$ largest eigenvalues are approximated, the associated eigenvectors can be obtained via

$$
\left|\tilde{\lambda}_{i}\right\rangle=V_{\boldsymbol{\beta}_{\mathrm{opt}}}^{\dagger}\left|z_{i}\right\rangle
$$

where $\left|z_{i}\right\rangle=X^{z_{i}} \otimes \cdots \otimes X^{z_{n}}|0\rangle^{\otimes n}$ and where $z_{i} \in\{0,1\}$.

\section{Computing TQFI bounds}

In this section we describe the variational quantum fidelity estimation (VQFE) algorithm of Ref. [45]. This algorithm is employed as a subroutine in VQFIE to estimate the TQFI bounds.

The input to VQFE is an $n$-qubit state $\rho_{\theta+\delta}$ and the estimates of the $m$ largest eigenvalues $\left(\left\{\tilde{\lambda}_{i}\right\}_{i=1}^{m}\right)$ and associated eigenvectors $\left(\left\{\left|\tilde{\lambda}_{i}\right\rangle\right\}_{i=1}^{m}\right)$ of $\rho_{\theta}$, both of which are obtained from VQSE. The goal of VQFE is to compute the generalized fidelity, which we recall for convenience:

$$
\begin{aligned}
F_{*}\left(\rho_{\theta}^{(m)}, \rho_{\theta+\delta}^{(m)}\right)= & \left\|\sqrt{\rho_{\theta}^{(m)}} \sqrt{\rho_{\theta+\delta}^{(m)}}\right\|_{1} \\
& +\sqrt{\left(1-\operatorname{Tr}\left[\rho_{\theta}^{(m)}\right]\right)\left(1-\operatorname{Tr}\left[\rho_{\theta+\delta}^{(m)}\right]\right)} .
\end{aligned}
$$

Here, we define the so-called $T$ matrix, whose elements are given by

$$
T_{i j}=\sqrt{\tilde{\lambda}_{i} \tilde{\lambda}_{j}}\left(\rho_{\theta+\delta}^{(m)}\right)_{i j}
$$

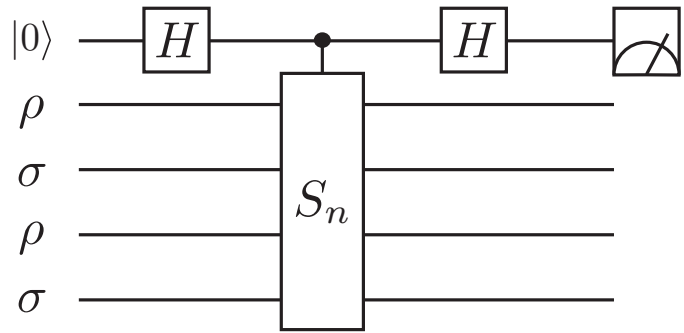

FIG. 6. The generalized SWAP test. Using one ancillary qubit to control a cyclic permutation gate, one can estimate linear and nonlinear functionals of a quantum state $[78,82]$.

where

$$
\left(\rho_{\theta+\delta}^{(m)}\right)_{i j}=\left\langle\tilde{\lambda}_{i}\left|\rho_{\theta+\delta}^{(m)}\right| \tilde{\lambda}_{j}\right\rangle .
$$

Note that the first term in the generalized fidelity can be expressed as

$$
\left\|\sqrt{\rho_{\theta}^{(m)}} \sqrt{\rho_{\theta+\delta}^{(m)}}\right\|_{1}=\operatorname{Tr} \sqrt{\sum_{i, j} T_{i j}\left|\tilde{\lambda}_{i}\right\rangle\left\langle\tilde{\lambda}_{j}\right|} .
$$

As described in Ref. [45], since we have access to the circuit $V_{\beta}^{\dagger}$ that prepares the estimated eigenvectors $\left|\tilde{\lambda}_{i}\right\rangle$, then the matrix elements $\left(\rho_{\theta+\delta}^{(m)}\right)_{i j}$ can be efficiently estimated in a quantum computer via a nonvariational algorithm. Hence, with postprocessing, one can always classically create and diagonalize the $m \times m T$ matrix to obtain (A8). This step is efficient as we assume that $m \in O[\operatorname{poly}(n)]$. Similarly, it is straightforward to see that the second term in the generalized fidelity is also completely determined by $\left\{\tilde{\lambda}_{i}\right\}_{i=1}^{m}$ and by $\left(\rho_{\theta+\delta}^{(m)}\right)_{i j}$ (both of which are known $\left.\forall i, j=1, \ldots, m\right)$ as

$$
\begin{aligned}
F_{*}\left(\rho_{\theta}^{(m)}, \rho_{\theta+\delta}^{(m)}\right)= & \operatorname{Tr} \sqrt{\sum_{i, j} T_{i j}\left|\tilde{\lambda}_{i}\right\rangle\left\langle\tilde{\lambda}_{j}\right|} \\
& +\sqrt{\left(1-\sum_{i} \tilde{\lambda}_{i}\right)\left(1-\sum_{i}\left(\rho_{\theta+\delta}^{(m)}\right)_{i i}\right)} .
\end{aligned}
$$

Hence both TQFI bounds can be obtained from the (known) terms in (A9).

\section{Computing SSQFI bounds}

Here, we show how to estimate functionals of the form $\operatorname{Tr}[\rho \sigma \rho \sigma]$, which are needed to compute the SSQFI bounds. The circuit in Fig. 6 can be used to estimate this quantity for $n$ qubit quantum states $\rho$ and $\sigma$. In general, linear and nonlinear functionals of quantum states can be directly estimated using what we refer to as the generalized SWAP test $[78,82]$.

Let $\rho$ and $\sigma$ be $n$-qubit quantum states. Then in the standard orthonormal basis for $n$ qubits, we can write the first and second copies of the states as

$$
\begin{aligned}
& \rho=\sum_{i} a_{i}|\boldsymbol{i}\rangle\left\langle\boldsymbol{i}\left|, \quad \sigma=\sum_{j} b_{j}\right| \boldsymbol{j}\right\rangle\langle\boldsymbol{j}|, \\
& \rho=\sum_{\boldsymbol{k}} c_{\boldsymbol{k}}|\boldsymbol{k}\rangle\left\langle\boldsymbol{k}\left|, \quad \sigma=\sum_{\boldsymbol{l}} d_{\boldsymbol{l}}\right| \boldsymbol{l}\right\rangle\langle\boldsymbol{l}|,
\end{aligned}
$$


where the boldface indices are bit strings of the form $i=$ $i_{1} i_{2} \cdots i_{n}$ with $i_{1}, i_{2}, \ldots, i_{n} \in\{0,1\}$. Thus the initial state in Fig. 6 is given as

$$
\rho_{\mathrm{in}}=\sum_{\boldsymbol{i}, \boldsymbol{j}, \boldsymbol{k}, \boldsymbol{l}} a_{i} b_{\boldsymbol{j}} c_{\boldsymbol{k}} d_{l}|0, \boldsymbol{i}, \boldsymbol{j}, \boldsymbol{k}, \boldsymbol{l}\rangle\langle 0, \boldsymbol{i}, \boldsymbol{j}, \boldsymbol{k}, \boldsymbol{l}| .
$$

The Hadamard gate transforms the standard basis vectors as

$$
\begin{aligned}
& H|0\rangle=\frac{1}{\sqrt{2}}(|0\rangle+|1\rangle), \\
& H|1\rangle=\frac{1}{\sqrt{2}}(|0\rangle-|1\rangle) .
\end{aligned}
$$

The cyclic shift operator (referred to as a permutation gate in the main text), $S_{n}$, is defined by its action on a tensor product basis for $n$-qubits:

$$
S_{n}\left|\psi_{1}, \psi_{2}, \ldots, \psi_{n-1}, \psi_{n}\right\rangle=\left|\psi_{n}, \psi_{1}, \psi_{2}, \ldots, \psi_{n-1}\right\rangle .
$$

Note that in the case of $n=2$, the cyclic shift operator is simply the familiar SWAP operator [85]. Thus the action of the gate sequence in Fig. 6 is

$$
|0, \boldsymbol{i}, \boldsymbol{j}, \boldsymbol{k}, \boldsymbol{l}\rangle
$$

$$
\begin{array}{cl}
\stackrel{\text { Hadamard }}{\longrightarrow} & \frac{1}{\sqrt{2}}(|0, \boldsymbol{i}, \boldsymbol{j}, \boldsymbol{k}, \boldsymbol{l}\rangle+|1, \boldsymbol{i}, \boldsymbol{j}, \boldsymbol{k}, \boldsymbol{l}\rangle), \\
\stackrel{\text { controlled- } S_{n}}{\longrightarrow} & \frac{1}{\sqrt{2}}(|0, \boldsymbol{i}, \boldsymbol{j}, \boldsymbol{k}, \boldsymbol{l}\rangle+|1, \boldsymbol{j}, \boldsymbol{k}, \boldsymbol{l}, \boldsymbol{i},\rangle), \\
\stackrel{\text { Hadamard }}{\longrightarrow} & \frac{1}{2}(|0, \boldsymbol{i}, \boldsymbol{j}, \boldsymbol{k}, \boldsymbol{l}\rangle+|0, \boldsymbol{j}, \boldsymbol{k}, \boldsymbol{l}, \boldsymbol{i},\rangle) .
\end{array}
$$

Recalling Eq. (A11), one can see that the probability of measuring the ancilla qubit in the $|0\rangle$ state is

$$
\begin{aligned}
p(0) & =\frac{1}{2}+\frac{1}{2} \sum_{i, \boldsymbol{j}, \boldsymbol{k}, \boldsymbol{l}} a_{i} b_{j} c_{\boldsymbol{k}} d_{\boldsymbol{l}}\langle 0, \boldsymbol{i}, \boldsymbol{j}, \boldsymbol{k}, \boldsymbol{l} \mid 0, \boldsymbol{j}, \boldsymbol{k}, \boldsymbol{l}, \boldsymbol{i}\rangle \\
& =\frac{1}{2}+\frac{1}{2} \sum_{i, \boldsymbol{j}, \boldsymbol{k}, \boldsymbol{l}} a_{i} b_{j} c_{\boldsymbol{k}} d_{\boldsymbol{l}} \delta_{i j} \delta_{j \boldsymbol{k}} \delta_{\boldsymbol{k} \boldsymbol{l}} \delta_{l i} \\
& =\frac{1}{2}+\frac{1}{2} \sum_{i} a_{i} b_{i} c_{i} d_{i}, \\
p(0) & =\frac{1}{2}+\frac{1}{2} \operatorname{Tr}[\rho \sigma \rho \sigma] .
\end{aligned}
$$

So, using a quantum computer with $4 n+1$ qubits, one can directly estimate the functional $\operatorname{Tr}[\rho \sigma \rho \sigma]$ using the probability of measuring the ancillary qubit in the zero state. Moreover, removing the second copies of $\rho$ and $\sigma$, one is able to estimate functionals of the form $\operatorname{Tr}[\rho \sigma]$ using the same method. In that case, only $2 n+1$ qubits are needed, and the cyclic shift operator simply becomes the SWAP operator.

\section{APPENDIX B: LOWER BOUND COMPARISON}

In this Appendix, we first compare the conditions under which the TQFI lower bound and the purity-loss bound of Refs. [69,70] can saturate their inequalities and be equal to the QFI. Then we present details of how the purity-loss bound was computed in our heuristics.

\section{Bound saturation}

As shown below, while the TQFI bounds can always saturate the bounds (and be efficiently computable) for states with low rank $r$, the purity-loss bounds can never saturate the inequality for $r \geqslant 3$. This implies that the VQFIE bounds can always be tighter for low-rank states with $r \geqslant 3$.

First, let us recall that the TQFI bounds can be saturated if $m$ is equal to the rank $r$ of the probe state. Moreover, since one can estimate the truncated fidelities for $m \in O[\operatorname{poly}(n)]$, this means that the TQFI bounds can be saturated for low-rank states with $r \in O[\operatorname{poly}(n)]$.

Let us now analyze the purity-loss bound. In the main text we defined the quantity

$$
\begin{aligned}
\mathcal{L}\left(\theta ; \rho_{\theta}\right) & =4\left(\operatorname{Tr}\left[\rho^{2} G^{2}\right]-\operatorname{Tr}[\rho G \rho G]\right) \\
& =2 \sum_{i, j}\left(\lambda_{i}-\lambda_{j}\right)^{2}\left|\left\langle\lambda_{i}|G| \lambda_{j}\right\rangle\right|^{2},
\end{aligned}
$$

which is a lower bound on the QFI as $\mathcal{L}\left(\theta ; \rho_{\theta}\right) \leqslant I\left(\theta ; \rho_{\theta}\right)$. Then, from the definition of the quantum Fisher information $[32,33]$, we have

$$
I\left(\theta ; \rho_{\theta}\right)=2 \sum_{i, j} \frac{\left(\lambda_{i}-\lambda_{j}\right)^{2}}{\lambda_{i}+\lambda_{j}}\left|\left\langle\lambda_{i}|G| \lambda_{j}\right\rangle\right|^{2} .
$$

From the fact that

$$
\frac{1}{\lambda_{i}+\lambda_{j}} \geqslant 1
$$

it is easy to see that $I\left(\theta ; \rho_{\theta}\right)$ takes its minimum when

$$
\lambda_{i}+\lambda_{j}=1 .
$$

The condition in (B4) implies that the QFI reaches its minima when $\rho_{\theta}$ is either a rank-1 or a rank-2 state. Hence it is straightforward to see that if (B4) holds, we have

$$
I\left(\theta ; \rho_{\theta}\right)=\mathcal{L}\left(\theta ; \rho_{\theta}\right) .
$$

However, for $r \geqslant 3$, the lower bound cannot saturate the inequality, so that we have the following strict inequality:

$$
I\left(\theta ; \rho_{\theta}\right)>\mathcal{L}\left(\theta ; \rho_{\theta}\right) \quad \text { when } r \geqslant 3 .
$$

\section{Heuristical computation of the purity loss}

In this section we describe additional details of how $\rho_{\text {ave }}$ was computed. As shown in Ref. [69] and as described in the main text, the lower bound in (B1) can be approximated by

$$
\mathcal{L}\left(\theta ; \rho_{\theta}\right) \approx 2 \frac{\Delta v}{(\Delta x)^{2}},
$$

where

$$
\Delta v=\operatorname{Tr}\left[\rho^{2}\right]-\operatorname{Tr}\left[\rho_{\text {ave }}^{2}\right]
$$

is the purity loss and where $(\Delta x)^{2}$ is the variance of the random variable $\theta(x)$, which defines the statistical fluctuation in the source. In Ref. [70], the authors proposed computing the state $\rho_{\text {ave }}$ by using a stratified sampling technique. Namely, they assumed that $\theta(x)$ is drawn from a discretized Gaussian distribution $\mathcal{G}$ with $K$ samples (or strata) and a variance $(\Delta x)^{2}$. 
That is,

$$
\rho_{\mathrm{ave}} \approx \frac{1}{K} \sum_{j=1}^{K} \rho_{\theta_{j}}
$$

where $\rho_{\theta_{j}}=W_{\theta_{j}} \rho W_{\theta_{j}}^{\dagger}$ and where $\theta_{j}$ is taken from $\mathcal{G}$. Hence the purity of $\rho_{\text {ave }}$ can be expressed as

$$
\operatorname{Tr}\left[\rho_{\text {ave }}^{2}\right] \approx \frac{1}{K^{2}} \sum_{j=1}^{K} \operatorname{Tr}\left[\rho_{\theta_{j}}^{2}\right]+\frac{2}{K^{2}} \sum_{j<m}^{K} \operatorname{Tr}\left[\rho_{\theta_{j}} \rho_{\theta_{m}}\right]
$$

and can be efficiently computed via $\left(K^{2}+K\right) / 2$ destructive SWAP tests [81] between the states $\rho_{\theta_{j}}$ and $\rho_{\theta_{m}}$ for $j \geqslant m$.

As discussed in Ref. [70], the higher the number of strata $K$, the better the approximation in (B7). Hence in order to determine how many strata we use in our numerics, we here propose to determine $K$ so that the number of calls to a quantum computer is the same when computing the TQFI lower bound as that when computing the purity-loss bound.

First, let us determine how many calls to a quantum computer are necessary when computing the TQFI. As previously outlined, each iteration of VQFIE variationally diagonalizes the probe state and usually requires the use of a gradient descent algorithm over $p$ parameters in $V_{\beta}$. By employing the parameter shift rule $[86,87]$, this requires us to run $2 p$ quantum circuits. Moreover, the estimation of each gradient to a precision of $1 / \sqrt{s}$ requires $s$ shots. In our numerical implementation, the training algorithm in VQSE took $t=$ 200. Hence, in each iteration of VQFIE where the subroutine VQSE is run, we require $2 s t p$ calls to a quantum computer. Finally, we remark that to guarantee the trainability of VQSE, we assume a hardware-efficient Ansatz with $\log _{2}(n)$ layers, meaning that $p=n \log _{2}(n)$. Then, as outlined in Ref. [45], the fidelity computation requires one to estimate $m$ eigenvalues up to a precision of $1 / \sqrt{s}$. A conservative estimate assumes that $m=n$ eigenvalues are kept in the truncated state. Finally, as described in the previous section, computing the TQFI bounds requires the estimation of the matrix elements of the $m \times m$ symmetric matrix $T$ of Eq. (A6) up to a precision of $1 / \sqrt{s}$. Here, we recall that there are $\left(m^{2}+m\right) / 2$ independent matrix elements in a symmetric matrix of size $m \times m$. Hence, in total, VQFIE requires

\section{TQFI bound calls to quantum computer}

$$
=s\left(2 t n \log _{2}(n)+n+\frac{n+n^{2}}{2}\right) \text {. }
$$

We turn now to the purity-loss bound. This bound requires the estimation of $\operatorname{Tr}\left[\rho^{2}\right]$ to a precision of $1 / \sqrt{s}$ and also implies the computation of $\operatorname{Tr}\left[\rho_{\text {ave }}^{2}\right]$. As previously discussed, computing the purity of $\rho_{\text {ave }}$ requires estimating $\left(K^{2}+K\right) / 2$ state overlaps up to precision $1 / \sqrt{s}$, where $K$ is the number of strata used for $\rho_{\text {ave }}$. Hence the total number of calls to the quantum computer will be

Purity bound calls to quantum computer $=s\left(\frac{K^{2}+K}{2}+1\right)$.

Finally, the number of strata $K$ which leads to a fair comparison between the TQFI bound and the purity-loss bound can be found by numerically solving

$$
\frac{K^{2}+K}{2}+1=2 \operatorname{tn} \log _{2}(n)+n+\frac{n+n^{2}}{2} .
$$

[1] L. Pezzé and A. Smerzi, Entanglement, Nonlinear Dynamics, and the Heisenberg Limit, Phys. Rev. Lett. 102, 100401 (2009).

[2] V. Giovannetti, S. Lloyd, and L. Maccone, Advances in quantum metrology, Nat. Photonics 5, 222 (2011).

[3] C. L. Degen, F. Reinhard, and P. Cappellaro, Quantum sensing, Rev. Mod. Phys. 89, 035002 (2017).

[4] S. Bhattacharjee, U. Bhattacharya, W. Niedenzu, V. Mukherjee, and A. Dutta, Quantum magnetometry using two-stroke thermal machines, New J. Phys. 22, 013024 (2020).

[5] J. M. Taylor, P. Cappellaro, L. Childress, L. Jiang, D. Budker, P. R. Hemmer, A. Yacoby, R. Walsworth, and M. D. Lukin, High-sensitivity diamond magnetometer with nanoscale resolution, Nat. Phys. 4, 810 (2008).

[6] A. Sone, Q. Zhuang, and P. Cappellaro, Quantifying precision loss in local quantum thermometry via diagonal discord, Phys. Rev. A 98, 012115 (2018).

[7] A. Sone, Q. Zhuang, C. Li, Y.-X. Liu, and P. Cappellaro, Nonclassical correlations for quantum metrology in thermal equilibrium, Phys. Rev. A 99, 052318 (2019).

[8] L. A. Correa, M. Mehboudi, G. Adesso, and A. Sanpera, Individual Quantum Probes for Optimal Thermometry, Phys. Rev. Lett. 114, 220405 (2015).

[9] A. De Pasquale, D. Rossini, R. Fazio, and V. Giovannetti, Local quantum thermal susceptibility, Nat. Commun. 7, 12782 (2016).
[10] S. Lloyd, Enhanced sensitivity of photodetection via quantum illumination, Science 321, 1463 (2008).

[11] Q. Zhuang, Z. Zhang, and J. H. Shapiro, Optimum Mixed-State Discrimination for Noisy Entanglement-Enhanced Sensing, Phys. Rev. Lett. 118, 040801 (2017).

[12] Q. Zhuang, J. Preskill, and L. Jiang, Distributed quantum sensing enhanced by continuous-variable error correction, New J. Phys. 20, 022001 (2020).

[13] T. J. Proctor, P. A. Knott, and J. A. Dunningham, Multiparameter Estimation in Networked Quantum Sensors, Phys. Rev. Lett. 120, 080501 (2018).

[14] D. Burgarth and K. Yuasa, Quantum System Identification, Phys. Rev. Lett. 108, 080502 (2012).

[15] J. Zhang and M. Sarovar, Quantum Hamiltonian Identification from Measurement Time Traces, Phys. Rev. Lett. 113, 080401 (2014).

[16] D. Burgarth and K. Maruyama, Indirect Hamiltonian identification through a small gateway, New J. Phys. 11, 103019 (2009).

[17] A. Sone and P. Cappellaro, Hamiltonian identifiability assisted by single-probe measurement, Phys. Rev. A 95, 022335 (2017).

[18] C. Di Franco, M. Paternostro, and M. S. Kim, Hamiltonian Tomography in an Access-Limited Setting without State Initialization, Phys. Rev. Lett. 102, 187203 (2009).

[19] Y. Wang, D. Dong, A. Sone, I. R. Petersen, H. Yonezawa, and P. Cappellaro, Quantum Hamiltonian identifiability via a 
similarity transformation approach and beyond, IEEE Trans. Autom. Control 65, 4632 (2020).

[20] Y. Kato and N. Yamamoto, Structure identification and state initialization of spin networks with limited access, New J. Phys. 16, 023024 (2014).

[21] A. Sone and P. Cappellaro, Exact dimension estimation of interacting qubit systems assisted by a single quantum probe, Phys. Rev. A 96, 062334 (2017).

[22] M. Owari, K. Maruyama, T. Takui, and G. Kato, Probing an untouchable environment for its identification and control, Phys. Rev. A 91, 012343 (2015).

[23] A. Ajoy, Y.-X. Liu, K. Saha, L. Marseglia, J.-C. Jaskula, U. Bissbort, and P. Cappellaro, Quantum interpolation for highresolution sensing, Proc. Natl Acad. Sci. USA 114, 2149 (2017).

[24] I. Lovchinsky, A. O. Sushkov, E. Urbach, N. P. de Leon, S. Choi, K. De Greve, R. Evans, R. Gertner, E. Bersin, C. Müller, L. McGuinness, F. Jelezko, R. L. Walsworth, H. Park, and M. D. Lukin, Nuclear magnetic resonance detection and spectroscopy of single proteins using quantum logic, Science 351, 836 (2016).

[25] J. Choi, H. Zhou, R. Landig, H.-Y. Wu, X. Yu, S. V. Stetina, G. Kucsko, S. Mango, D. Needleman, A. D. T. Samuel, P. Maurer, H. Park, and M. D. Lukin, Probing and manipulating embryogenesis via nanoscale thermometry and temperature control, Proc. Natl. Acad. Sci. USA 117, 14636 (2020).

[26] M. Fujiwara, S. Sun, A. Dohms, Y. Nishimura, K. Suto, Y. Takezawa, K. Oshimi, L. Zhao, N. Sadzak, Y. Umehara, Y. Teki, N. Komatsu, O. Benson, Y. Shikano, and E. Kage-Nakadai, Real-time nanodiamond thermometry probing in-vivo thermogenic responses, Sci. Adv. 6, eaba9636 (2020).

[27] C. S. Wolfe, S. A. Manuilov, C. M. Purser, R. Teeling-Smith, C. Dubs, P. C. Hammel, and V. P. Bhallamudi, Spatially resolved detection of complex ferromagnetic dynamics using optically detected nitrogen-vacancy spins, Appl. Phys. Lett. 108, 232409 (2016).

[28] F. Casola, T. van der Sar, and A. Yacoby, Probing condensed matter physics with magnetometry based on nitrogen-vacancy centres in diamond, Nat. Rev. Mater. 3, 17088 (2018).

[29] S. Rajendran, N. Zobrist, A. O. Sushkov, R. Walsworth, and M. Lukin, A method for directional detection of dark matter using spectroscopy of crystal defects, Phys. Rev. D 96, 035009 (2017).

[30] L. McCuller, C. Whittle, D. Ganapathy, K. Komori, M. Tse, A. Fernandez-Galiana, L. Barsotti, P. Fritschel, M. MacInnis, F. Matichard, K. Mason, N. Mavalvala, R. Mittleman, H. Yu, M. E. Zucker, and M. Evans, Frequency-Dependent Squeezing for Advanced LIGO, Phys. Rev. Lett. 124, 171102 (2020).

[31] B. P. Abbott et al. (LIGO Scientific Collaboration and Virgo Collaboration), Observation of Gravitational Waves from a Binary Black Hole Merger, Phys. Rev. Lett. 116, 061102 (2016).

[32] M. Hayashi, Quantum Information Theory: Mathematical Foundation, 2nd ed. (Springer, New York, 2004).

[33] J. Liu, H. Yuan, X.-M. Lu, and X. Wang, Quantum Fisher information matrix and multiparameter estimation, J. Phys. A: Math. Theor. 53, 023001 (2020).

[34] A. Fujiwara and H. Nagaoka, Quantum Fisher metric and estimation for pure state models, Phys. Lett. A 201, 119 (1995).
[35] K. Matsumoto, A new approach to the Cramér-Rao-type bound of the pure-state model, J. Phys. A: Math. Gen. 35, 3111 (2002).

[36] L. J. Fiderer, J. M. E. Fraïsse, and D. Braun, Maximal Quantum Fisher Information for Mixed States, Phys. Rev. Lett. 123, 250502 (2019).

[37] J. Preskill, Quantum computing in the NISQ era and beyond, Quantum 2, 79 (2018).

[38] M. Cerezo, A. Arrasmith, R. Babbush, S. C. Benjamin, S. Endo, K. Fujii, J. R. McClean, K. Mitarai, X. Yuan, L. Cincio, and P. J. Coles, Variational quantum algorithms, Nat. Rev. Phys. 3, 625 (2021).

[39] A. Peruzzo, J. McClean, P. Shadbolt, M.-H. Yung, X.-Q. Zhou, P. J. Love, A. Aspuru-Guzik, and J. L. O'Brien, A variational eigenvalue solver on a photonic quantum processor, Nat. Commun. 5, 4213 (2014).

[40] E. Farhi, J. Goldstone, and S. Gutmann, A quantum approximate optimization algorithm, arXiv:1411.4028.

[41] J. Romero, J. P. Olson, and A. Aspuru-Guzik, Quantum autoencoders for efficient compression of quantum data, Quantum Sci. Technol. 2, 045001 (2017).

[42] S. Khatri, R. LaRose, A. Poremba, L. Cincio, A. T Sornborger, and P. J. Coles, Quantum-assisted quantum compiling, Quantum 3, 140 (2019).

[43] R. LaRose, A. Tikku, É. O’Neel-Judy, L. Cincio, and P. J. Coles, Variational quantum state diagonalization, npj Quantum Inf. 5, 57 (2019); 5, 67(E) (2019).

[44] A. Arrasmith, L. Cincio, A. T. Sornborger, W. H. Zurek, and P. J. Coles, Variational consistent histories as a hybrid algorithm for quantum foundations, Nat. Commun. 10, 3438 (2019).

[45] M. Cerezo, A. Poremba, L. Cincio, and P. J. Coles, Variational quantum fidelity estimation, Quantum 4, 248 (2020).

[46] K. Sharma, S. Khatri, M. Cerezo, and P. J. Coles, Noise resilience of variational quantum compiling, New J. Phys. 22, 043006 (2020).

[47] C. Bravo-Prieto, D. García-Martín, and J. I. Latorre, Quantum singular value decomposer, Phys. Rev. A 101, 062310 (2020).

[48] M. Cerezo, K. Sharma, A. Arrasmith, and P. J. Coles, Variational quantum state eigensolver, arXiv:2004.01372.

[49] C. Cirstoiu, Z. Holmes, J. Iosue, L. Cincio, P. J. Coles, and A. Sornborger, Variational fast forwarding for quantum simulation beyond the coherence time, npj Quantum Inf. 6, 82 (2020).

[50] X. Yuan, S. Endo, Q. Zhao, Y. Li, and S. C. Benjamin, Theory of variational quantum simulation, Quantum 3, 191 (2019).

[51] H.-L. Huang, Y. Du, M. Gong, Y. Zhao, Y. Wu, C. Wang, S. Li, F. Liang, J. Lin, Y. Xu, R. Yang, T. Liu, M.-H. Hsieh, H. Deng, H. Rong, C.-Z. Peng, C.-Y. Lu, Y.-A. Chen, D. Tao, X. Zhu, and J.-W. Pan, Experimental Quantum Generative Adversarial Networks for Image Generation, Phys. Rev. Appl. 16, 024051 (2021).

[52] J. Carolan, M. Mohseni, J. P. Olson, M. Prabhu, C. Chen, D. Bunandar, M. Y. Niu, N. C. Harris, F. N. C. Wong, M. Hochberg, S. Lloyd, and D. Englund, Variational quantum unsampling on a quantum photonic processor, Nat. Phys. 16, 322 (2020).

[53] C. Bravo-Prieto, R. LaRose, M. Cerezo, Y. Subasi, L. Cincio, and P. J. Coles, Variational quantum linear solver: A hybrid algorithm for linear systems, arXiv:1909.05820. 
[54] E. Anschuetz, J. Olson, A. Aspuru-Guzik, and Y. Cao, Variational quantum factoring, in International Workshop on Quantum Technology and Optimization Problems (Springer, New York, 2019), pp. 74-85.

[55] J. R. McClean, S. Boixo, V. N. Smelyanskiy, R. Babbush, and H. Neven, Barren plateaus in quantum neural network training landscapes, Nat. Commun. 9, 4812 (2018).

[56] M. Cerezo, A. Sone, T. Volkoff, L. Cincio, and P. J. Coles, Cost function dependent barren plateaus in shallow parametrized quantum circuits, Nat. Commun. 12, 1791 (2021).

[57] K. Sharma, M. Cerezo, L. Cincio, and P. J. Coles, Trainability of dissipative perceptron-based quantum neural networks, arXiv:2005.12458.

[58] T. Volkoff and P. J. Coles, Large gradients via correlation in random parameterized quantum circuits, Quantum Sci. Technol. 6, 025008 (2021).

[59] S. Wang, E. Fontana, M. Cerezo, K. Sharma, A. Sone, L. Cincio, and P. J. Coles, Noise-induced barren plateaus in variational quantum algorithms, Nat. Commun. 12, 6961 (2021).

[60] M. Cerezo and P. J. Coles, Impact of barren plateaus on the Hessian and higher order derivatives, Quantum Sci. Technol. 6, 035006 (2021).

[61] Z. Holmes, A. Arrasmith, B. Yan, P. J. Coles, A. Albrecht, and A. T. Sornborger, Barren Plateaus Preclude Learning Scramblers, Phys. Rev. Lett. 126, 190501 (2021).

[62] A. Hentschel and B. C. Sanders, Machine Learning for Precise Quantum Measurement, Phys. Rev. Lett. 104, 063603 (2010).

[63] H. Xu, J. Li, L. Liu, Y. Wang, H. Yuan, and X. Wang, Generalizable control for quantum parameter estimation through reinforcement learning, npj Quantum Inf. 5, 82 (2019).

[64] T. Xiao, J. Huang, J. Fan, and G. Zeng, Continuous-variable quantum phase estimation based on machine learning, Sci. Rep. 9, 12410 (2019).

[65] R. Kaubruegger, P. Silvi, C. Kokail, R. van Bijnen, A. M. Rey, J. Ye, A. M. Kaufman, and P. Zoller, Variational Spin-Squeezing Algorithms on Programmable Quantum Sensors, Phys. Rev. Lett. 123, 260505 (2019).

[66] L. Pezzè, A. Smerzi, M. K. Oberthaler, R. Schmied, and P. Treutlein, Quantum metrology with nonclassical states of atomic ensembles, Rev. Mod. Phys. 90, 035005 (2018).

[67] B. Koczor, S. Endo, T. Jones, Y. Matsuzaki, and S. C. Benjamin, Variational-state quantum metrology, New J. Phys. 22, 083038 (2020).

[68] J. J. Meyer, J. Borregaard, and J. Eisert, A variational toolbox for quantum multi-parameter estimation, npj Quantum Inf. 7, 89 (2021).

[69] K. Modi, L. C. Céleri, J. Thompson, and M. Gu, Fragile states are better for quantum metrology, arXiv:1608.01443.

[70] X. Yang, J. Thompson, Z. Wu, M. Gu, X. Peng, and J. Du, Probe optimization for quantum metrology via closed-loop learning control, npj Quantum Inf. 6, 62 (2020).
[71] J. Liu, J. Chen, X.-X. Jing, and X. Wang, Quantum Fisher information and symmetric logarithmic derivative via anticommutators, J. Phys. A: Math. Theor. 49, 275302 (2016).

[72] D. Girolami, Observable Measure of Quantum Coherence in Finite Dimensional Systems, Phys. Rev. Lett. 113, 170401 (2014).

[73] C. Zhang, B. Yadin, Z.-B. Hou, H. Cao, B.-H. Liu, Y.-F. Huang, R. Maity, V. Vedral, C.-F. Li, G.-C. Guo, and D. Girolami, Detecting metrologically useful asymmetry and entanglement by a few local measurements, Phys. Rev. A 96, 042327 (2017).

[74] V. Katariya and M. M. Wilde, Geometric distinguishability measures limit quantum channel estimation and discrimination, Quantum Inf. Process. 20, 78 (2021).

[75] A. Sone, M. Cerezo, J. L. Beckey, and P. J. Coles, Generalized measure of quantum Fisher information, Phys. Rev. A 104, 062602 (2021).

[76] J. A. Miszczak, Z. Puchala, P. Horodecki, A. Uhlmann, and K. Zyczkowski, Sub- and super-fidelity as bounds for quantum fidelity, Quantum Inf. Comput. 9, 103 (2009).

[77] M. Tomamichel, Quantum Information Processing with Finite Resources: Mathematical Foundations, Springer Briefs in Mathematical Physics (Springer, New York, 2016), Vol. 5.

[78] A. K. Ekert, C. M. Alves, D. K. L. Oi, M. Horodecki, P. Horodecki, and L. C. Kwek, Direct Estimations of Linear and Nonlinear Functionals of a Quantum State, Phys. Rev. Lett. 88, 217901 (2002).

[79] M. Cerezo, A. Sone, J. L. Beckey, and P. J. Coles, Subquantum Fisher information, Quantum Sci. Technol. 6, 035008 (2021).

[80] A. Kandala, A. Mezzacapo, K. Temme, M. Takita, M. Brink, J. M. Chow, and J. M. Gambetta, Hardware-efficient variational quantum eigensolver for small molecules and quantum magnets, Nature (London) 549, 242 (2017).

[81] L. Cincio, Y. Subaş1, A. T. Sornborger, and P. J. Coles, Learning the quantum algorithm for state overlap, New J. Phys. 20, 113022 (2018).

[82] T. A. Brun, Measuring polynomial functions of states, Quantum Inf. Comput. 4, 401 (2004).

[83] M. J. D. Powell, The BOBYQA algorithm for bound constrained optimization without derivatives, Technical Report No. DAMTP 2009/NA06, Department of Applied Mathematics and Theoretical Physics, University of Cambridge, 2009.

[84] A. Arrasmith, Z. Holmes, M. Cerezo, and P. J. Coles, Equivalence of quantum barren plateaus to cost concentration and narrow gorges, arXiv:2104.05868.

[85] M. A. Nielsen and I. L. Chuang, Quantum Computation and Quantum Information: 10th Anniversary Edition, 10th ed. (Cambridge University Press, New York, 2011).

[86] K. Mitarai, M. Negoro, M. Kitagawa, and K. Fujii, Quantum circuit learning, Phys. Rev. A 98, 032309 (2018).

[87] M. Schuld, V. Bergholm, C. Gogolin, J. Izaac, and N. Killoran, Evaluating analytic gradients on quantum hardware, Phys. Rev. A 99, 032331 (2019). 